\title{
Localização de Pontos Fixos e Coincidências
}

\section{Daniel Vendrúscolo}

\author{
DISSERTAÇÃO APRESENTADA \\ $\mathrm{AO}$ \\ INSTITUTO DE MATEMÁTICA E ESTATÍSTICA \\ DA \\ UNIVERSIDADE DE SÃO PAULO \\ PARA \\ OBTENÇÃO DO GRAU DE MESTRE \\ EM \\ MATEMÁTICA
}

Área de Concentração: Topologia Algébrica Orientador: Prof. Dr. Daciberg Lima Gonçalves

Durante a elaboraçào deste trabalho o autor recebeu apoio financeiro do CNPq.

-São Paulo, Março de 1998- 


\section{Localização de Pontos Fixos e Coincidências}

Este exemplar corresponde à redação final da dissertação devidamente corrigida e defendida por Daniel Vendrúscolo e aprovada pela comissão julgadora.

São Paulo, 06 de Março de 1998.

Banca examinadora:

- Prof. Dr. Daciberg Lima Gonçalves (Orientador) - IME - USP

- Prof. Dra. Lucícia Daruiz Borsari - IME - USP

- Prof. Dr. Janey Antonio Daccach - ICAISC - USP. 


\section{Agradecimentos}

Ao Prof. Daciberg pela orientaçào precisa, cordial e indispensável.

À minha família, especialmente aos meus pais. por tudo.

Aos meus amigos do IME; Patrícia, Raul. Fernando, Walquíria, Samuel, Cecília. José Antônio, Irene e demais. pela companhia constante.

Aos fiéis amigos Bologna e Xirim em reconhecimento a um trabalho de persuasào muitas vezes imprescindivel.

Especialmente à Liane Bordignon por estar ao meu lado. 


\title{
Resumo
}

Nesse trabalho apresentamos alguns resultados de localização de pontos fixos em poliedros obtidos por Helga Schimer. Abordamos também os mesmos problemas para coincidências. enunciando-os, sempre que possivel. para complexos simpliciais e não apenas variedades.

Demonstramos que: (i) Todo poliedro do tipo $W$ tem a propriedade da invariância completa. (ii) Sejam $X^{\prime}$ e $Y n$-variedades $(n \geq 2)$, conexas, compactas, orientáveis, triangularizáveis e sem bordo; se $A \neq \emptyset$ é um fechado em $X$, dada $f: X \rightarrow Y$, existem $f_{1}, f_{2}$ homotópicas à $f \operatorname{com} C \operatorname{oin}\left(f_{1}, f_{2}\right)=A$. (iii) Respeitadas as condições de realização de $N(f)$ e $N\left(f_{1}, f_{2}\right)$ podemos realizar as classes de Nielsen essenciais como qualquer conjunto finito de pontos que satisfaçam as condiçòes dos índices das classes (no caso de coincidências as imagens das coincidências também podem ser qualquer conjunto). (iv) Seja $|Y|$ um poliedro compacto do tipo $W$, se $A \subset|Y|$ é um fechado entào podemos realizar $A$ como imagem do conjunto de coincidências de um par de aplicações de $\left|X^{\prime}\right|$ em $|Y|$ onde $\left|X^{\prime}\right|$ é um poliedro qualquer de mesma dimensão que $|Y|$.

\begin{abstract}
In this work we present several results about the localization of fixed points in polyhedra obtained by Helga Schimer. We approach the same problens in coincidence theory, enunciating them (whenever possible) for polyhedra and not only for manifolds.

We show that: (i) All polyhedron of type $W$ have the complete invariance property. (ii) Let $\mathrm{X}$ and $Y$ be two orientable connected compact triangulable $n$-manifold without boundary, if $A \neq \emptyset$ is closed on $X$ and $f: X \rightarrow Y$ is a map, then there are $f_{1}, f_{2}$ which are homotopics to $f$ with $\operatorname{Coin}\left(f_{1}, f_{2}\right)=A$. (iii) Under sufficient conditions for the realization of $N(f)$ and $N\left(f_{1}, f_{2}\right)$ we can realize the essencial Nielsen classes like any finite set of points which satisfy the conditions of indices of classes (for coincidences the ranges can be any set). (iv) Let $|Y|$ a compact polihedron of type $\mathrm{W}$. if $A \subset|Y|$ is closed. then there are $f_{1}, f_{2}:\left|X^{\prime}\right| \rightarrow|Y|$ such that $f_{i}\left(\operatorname{Coin}\left(f_{1}, f_{2}\right)\right)=A$ and $\left|X^{\prime}\right|$ is any polihedron of the same dimension of $|Y|$.
\end{abstract}




\section{Conteúdo}

1 Preliminares 3

1.1 Índice de Ponto Fixo . . . . . . . . . . . . . . . . . . . 3

1.2 Número de Nielsen . . . . . . . . . . . . . . . . . 7

1.3 Índice de Coincidências . . . . . . . . . . . . . . . . . . . . 11

1.4 Número de Lefschetz . . . . . . . . . . . . . . . . . . . . 15

1.5 Número de Nielsen para Coincidência . . . . . . . . . . . . . . 18

2 Localização de Pontos Fixos $\quad 21$

3 Localização de Coincidências $\quad 36$

$\begin{array}{ll}\text { Bibliografia } & 46\end{array}$ 


\section{Introdução}

O objetivo desse trabalho é apresentar resultados de localização de pontos fixos e coincidências no contexto da teoria de Nielsen.

No primeiro capítulo fazemos uma exposição da teoria de Nielsen para pontos fixos e coincidências. Usamos como referências os livros [Jiang], [Kiang] e [Vick] além da tese [Oliveira].

No capítulo dois, que se baseia nos artigos [Schimer 1] e [Schimer 2], demonstramos alguns resultados de localização de pontos fixos. Entre os teoremas apresentados destacamos: 2.9, 2.18 (enunciados para deformações), 2.21 e 2.22, esse último para a esfera $S^{2}$. O livro [Brown] torna-se, aqui, uma referência indispensável pois contém quase toda a técnica utilizada além dos teoremas básicos de realização.

O terceiro capítulo é dedicado à localização de coincidências. Utilizando [Schimer 3] obtemos resultados semelhantes aos apresentados para pontos fixos, onde destacam-se os teoremas 3.6 (deformação) e 3.8. Ainda no contexto de coincidências, analisando a localização das imagens das coincidências, demonstramos os teoremas 3.10 e 3.12 (deformação). Apesar de quase todos os principais teoremas estarem enunciados para variedades, onde a teoria de coincidências esta mais consolidada, procuramos. sempre que possivel, obter lemas que possam ser utilizados no contexto mais geral de poliedros. Dessa forma as técnicas utilizadas nesse capítulo não são muito distintas das do capítulo anterior.

Finalmente gostaríamos de salientar que as demonstrações que podem ser encontradas nos livros da bibliografia foram omitidas. mas apresentamos. com detalhes, quase todas as demonstrações só disponiveis nos artigos. 


\section{Capítulo 1}

\section{Preliminares}

A idéia central desse capítulo é descrever a teoria básica de coincidência e de ponto fixo.

\section{1 Índice de Ponto Fixo}

Definição 1.1 Dada uma função $f: X \rightarrow X$ dizemos que $x \in X$ é um Ponto Fixo da $f$ se $f(x)=x$.

Denotaremos por Fix $(f)$ o conjunto dos pontos fixo da função $f$.

Vamos inicialmente definir um índice de pontos fixos para aplicaçǒes de $\mathbb{R}^{n} \mathrm{em}$ $\mathbb{R}^{n}$ de forma semelhante a feita em [Dold 2].

Definição 1.2 Seja $f: U \rightarrow \mathbb{R}^{n} ; U \subset \mathbb{R}^{n}$ aberto e $W \subseteq U$ aberto com Fix $(f) \cap W$ compacto; tomamos $V \subseteq W$ tal que: $F i x(f) \cap W \subset V \subseteq \bar{V} \subseteq W$ denotando por $o_{n}$ a classe fundamental de $H_{n}(W, W \backslash V ; \mathbb{Q})$ chamamos de índice de $f$ em $W, I_{f}^{W}$, o inteiro:

$$
(i d-f)_{*}\left(o_{n}\right) \in H_{n}\left(\mathbb{R}^{n} \cdot \mathbb{R}^{n} \backslash 0\right) \approx \mathbb{Z}
$$

onde id é a aplicação identidade.

Se $W=U$ denotamos $I_{f}^{W}$ por $I_{f}$.

O índice de pontos fixos satisfaz as seguintes propriedades (para maiores detalhes ver [Dold 2. pp 202:205]): 


\section{Localização}

Sejam $f . f^{\prime}$ como na definiçào. $W^{\prime}$ e $W^{\prime \prime}$ abertos tais que $F i x(f) \cap W^{*}=F i r\left(f^{\prime}\right) \cap$ $W^{\prime}$ e $\left.f\right|_{W \cap \cap W^{\prime}}=\left.f^{\prime}\right|_{W \cap W^{\prime}}$ entào:

$$
I_{f}^{I V}=I_{f^{\prime}}^{I^{\prime \prime}}
$$

Demonstração: Como $\left.f\right|_{W \cap W^{\prime}}=\left.f^{\prime}\right|_{W \cap W^{\prime}}$ temos, claramente $(i d-f)_{\times}=$ $\left(i d-f^{\prime}\right)_{\times} ; \operatorname{assim} I_{f}^{W}$ depende exclusivamente de $\left.f\right|_{W}$.

\section{Aditividade}

Sejam $f$ e $W$ como na definição; se $W$ é tal que $W=\bigcup_{i=1}^{k} W_{i} \operatorname{com} W_{i} \cap W_{j}=\emptyset$ se $i \neq j$ então:

$$
I_{f}^{W}=\sum_{i=1}^{k} I_{f}^{W_{i}}
$$

Demonstração: Tomando $V=\bigcup_{i=1}^{k} V_{i}$ tal que $F i x(f) \cap W_{i} \subset V_{i} \subseteq \overline{V_{i}} \subseteq W_{i}$ temos que $H_{n}(W, W \backslash V ; \mathbb{Q})=\bigoplus_{i} \stackrel{i=1}{H}_{n}\left(W_{i}, W_{i} \backslash V_{i} ; \mathbb{Q}\right)$, portanto:

$$
(i d-f)_{*}\left(o_{n}\right)=\sum_{i=1}^{k}(i d-f)_{*}\left(o_{n_{i}}\right)=\sum_{i=1}^{k} I_{f}^{W_{i}}
$$

onde cada $o_{n_{i}}$ é a classe fundamental de $H_{n}\left(W_{i}, W_{i} \backslash V_{i} ; \mathbb{Q}\right)$.

\section{Invariância Homotópica}

Se $H(x, t): U \times I \rightarrow \mathbb{R}^{n}$ é uma homotopia tal que $H(x, 0)=f$ e $H(x, 1)=g$ e se $\bigcup_{t} F i x(H(x, t)) \cap W$ é compacto entào:

$$
I_{f}^{W}=I_{g}^{W}
$$

Demonstração: Decorre facilmente do fato que $(i d-f)_{\times}=(i d-g)_{\times}$pois $i d-H(x . t)$ é uma homotopia entre $i d-f$ e $i d-g$.

\section{Multiplicatividade}

Se $f: l \rightarrow \mathbb{R}^{n} . g: U^{\prime} \rightarrow \mathbb{R}^{n}$, W e $W^{\prime \prime}$ são como na definçào $\left(W \subset U\right.$ e $\left.W^{\prime} \subset L^{\prime \prime}\right)$ então:

$$
I_{f \times g}^{W \times W^{\prime \prime}}=I_{f}^{W} \cdot I_{g}^{W^{\prime}}
$$


Demonstração: Se $o_{n}$ e $o_{n}^{\prime}$ são classes fundamentais de $H_{n}(W, \mathbb{W} \backslash V ; \mathbb{Q})$ e $H_{n}\left(W^{\prime} . W^{\prime \prime} \backslash V^{\prime}: \mathbb{Q}\right)$ respectivamente sabemos que $o_{n} \times o_{n}^{\prime}$ é a classe fundamental que nos interessa para calcular $I_{f \times g}^{W \times W^{\prime \prime}}$ : desse modo temos:

$$
\begin{aligned}
I_{f \times g}^{W \times W^{\prime}} & =(i d \times i d-f \times g)_{\times}\left(o_{n} \times o_{n}^{\prime}\right) \\
& =[(i d-f) \times(i d-g)]_{\times}\left(o_{n} \times o_{n}^{\prime}\right) \\
& =\left[(i d-f)_{\times}\left(o_{n}\right)\right] \times\left[(i d-g)_{\times}\left(o_{n}^{\prime}\right)\right] \\
& =I_{f}^{W} \cdot I_{g}^{W^{\prime}}
\end{aligned}
$$

\section{Comutatividade}

Se $f: U \rightarrow \mathbb{R}^{n}, g: U^{\prime} \rightarrow \mathbb{R}^{n^{\prime}}, W$ e $W^{\prime}$ são como na definção ( $W \subset U$ e $\left.W^{\prime} \subset U^{\prime}\right)$ tomando $V=f^{-1}\left(W^{\prime}\right)$ e $V^{\prime}=g^{-1}\left(W^{*}\right)$ temos:

$$
I_{g \circ f}^{V}=I_{f \circ g}^{V^{\prime}}
$$

Demonstração: É interessante notar que $F i x(g \circ f) \cap V$ é homeomorfo a Fix $(f \circ g) \cap V^{\prime}$. Definimos:

$$
\gamma: V \times V^{\prime} \rightarrow \mathbb{R}^{n} \times \mathbb{R}^{n^{\prime}}, \quad \gamma(x, y)=(g(y), f(x))
$$

Fazendo: $H: V \times V^{\prime} \times I: \rightarrow \mathbb{R}^{n} \times \mathbb{R}^{n^{\prime}}$ tal que

$$
H(x, y, t)=[t \cdot g \circ f(x)+(1-t) \cdot g(y), f(x)],
$$

temos que, para $t_{0}$ fixado,

$$
(x, y) \in F i x\left(H\left(x, y, t_{0}\right)\right) \Leftrightarrow y=f(x) \text { e } x \in F i x(g \circ f) .
$$

Assim Fix $\left.H\left(x, y, t_{0}\right)\right)$ independe de $t$ e é compacto. dessa forma, usando a propriedade da invariância homotópica, pois $\bigcup_{t} F i x(H(x, y, t))$ é compacto, temos que:

$$
I_{\gamma}^{V \times V^{\prime \prime}}=I_{H(x, y, 0)}^{V \times V^{\prime}}=I_{H(x, y, 1)}^{V \times V^{\prime \prime}} .
$$

Observamos que $H(x, y, 1)=(g \circ f(x), f(x))$ e assim:

$$
I_{H(x, y, 1)}^{V \times V^{\prime}}=I_{S}^{V \times V^{\prime \prime}}
$$

onde $\delta: V \times \mathbb{R}^{n^{\prime}} \rightarrow \mathbb{R}^{n} \times \mathbb{R}^{n^{\prime}}$ é dacla por $\delta(x, y)=(g \circ f(x) . f(x))$ (essa igualdade decorre da propriedade de localizaçào do índice de ponto fixo). 
Vamos agora deformar $\delta$ para obtermos $I_{\delta}^{V \times V^{\prime \prime}}=I_{g \circ f}^{V}$. Para tanto definimos a homotopia:

$$
G(x, y, t)=(g \circ f(x) \cdot(1-t) \cdot f(x))
$$

e observamos que $C_{r}(x, y, 1)=(g \circ f, 0)$. Assim podemos entender $C_{r}(x, y, 1)$ como uma aplicação produto $g \circ f \times$ cte $: V \times \mathbb{R}^{n^{\prime}} \rightarrow \mathbb{R}^{n} \times \mathbb{R}^{n^{\prime}}$, (cte a aplicaçào constante) e, usando a propriedade multiplicativa do índice, temos:

$$
I_{\delta}^{V \times V^{\prime \prime}}=I_{G(x, y, 0)}^{V^{\prime} \times V^{\prime}}=I_{G(x, y, 1)}^{V \times V^{\prime}}=I_{g \circ f}^{V} \cdot I_{c t e}^{V^{\prime}} .
$$

Como $(i d-c t e)_{*}=i d$, sabemos que $I_{c t e}^{V^{\prime}}=1$. Logo:

$$
I_{\sim_{i}}^{V^{\prime} \times V^{\prime \prime}}=I_{S}^{V \times V^{\prime}}=I_{g \circ f}^{V} .
$$

Repetindo o processo com $H^{\prime}(x, y, t)=(g(y), t \cdot f \circ g(y)+(1-t) \cdot f(x))$ e $G^{\prime}(x, y, t)=$ $((1-t) \cdot g(y), f \circ g(y))$ obtemos $I_{\gamma}^{V \times V^{\prime}}=I_{f \circ g}^{V^{\prime}}$.

Em [Dold 1], usando a propriedade comutativa descrita acima, temos a definiçào de índice em situações mais amplas.

Definição 1.3 Um espaço topológico $X$ é dito um $\mathbf{E N R}^{1}$ se existe $X^{\prime} \in \mathbb{R}^{n}$, retrato de vizinhança, homeomorfo a $X$.

São exemplos de ENR: os $\mathbb{R}^{n}$, abertos de $\mathbb{R}^{n}$, as variedades topológicas e os poliedros compactos.

Tomando $f: X \rightarrow X$ com $X$ um ENR podemos "decompor" $f$ na forma

$$
X \stackrel{i}{\rightarrow} U^{\prime} \stackrel{\text { for }}{\longrightarrow} X
$$

onde $i$ é o homeomorfismo entre $X$ e $X^{\prime} \operatorname{com} X^{\prime} \subset U^{\prime} \subseteq \mathbb{R}^{n}, U^{\prime}$ aberto e $r$ a retração de $U^{\prime}$ em $X^{\prime}$.

Observamos que se $U^{\prime \prime}$ é outro aberto de $\mathbb{R}^{n^{\prime}} \operatorname{com} X^{\prime \prime}$ homeomorfo a $X$ a propriedade comutativa nos garante que:

$$
I_{i \circ f \circ r}^{U^{\prime}}=I_{i \prime \prime}^{U^{\prime \prime} f \circ r^{\prime \prime}}
$$

onde $i^{\prime \prime}$ e $r^{\prime \prime}$ são os correspondentes de $i$ e de $r$.

Após essas consideraçòes podemos dar a seguinte definiçào:

\footnotetext{
${ }^{1}$ Sigla derivada da língua inglesa Euclidean Neighborhood Retract.
} 
Definição 1.4 Seja $f: X \rightarrow X$. X um ENR $\in \mathrm{X} \subseteq X$ aberto com Fix $(f) \cap \mathrm{I}^{\circ}$ compacto: definimos o índice de $f \mathrm{em} W$ satisfizendo:

$$
I_{f}^{W}=I_{i \circ f \circ r}^{r^{-1}(W)} .
$$

$I_{f}^{W}$ esta bem definido pelo exposto acima e as propriedades demonstradas anteriormente também sào verdadeiras com demonstrações muito semelhantes às que foram dadas na definição anterior.

Se $K \subset$ Fix $(f)$ é um compacto de $X$, tomando $W$ um aberto tal que $W \cap F i x(f)=$ $K$, denotaremos, por simplicidade,

$$
\operatorname{ind}(f, K)=I_{f}^{W} .
$$

Observamos que pela propriedade de localizaçào ind $(f, K)$ independe de $W$. Diremos. por abuso de linguagem índice da $f$ em $K$.

\subsection{Número de Nielsen}

Nessa secção vamos apresentar, resumidamente, as definições e principais resultados da teoria de classes de ponto fixo de Nielsen.

Sejam $X$ um poliedro compacto e $p: \widetilde{X} \rightarrow X_{\widetilde{X}}$ seu recobrimento universal. Denotando por $\mathfrak{D}(X)$ o grupo das aplicações $\gamma: \widetilde{X} \rightarrow \widetilde{X}$ tais que $\gamma \circ p=p \circ$ id. temos:

Definição 1.5 Dois levantamentos $\tilde{f}$ e $\tilde{f}^{\prime}$ de $f: X^{\prime} \rightarrow X^{\prime}$ são conjugados se existe $\gamma \in \mathfrak{D}\left(X^{\prime}\right)$ tal que $\widetilde{f}^{\prime}=\gamma \circ \widetilde{f} \circ \gamma^{-1}$.

Claramente a relação de conjugação é uma relaçào de equilavência.

Definição 1.6 Chamamos de classes de levantamento da $f$ as classes de equivalência dos levantamentos da f por conjugaçào, ou seja:

$$
[\tilde{f}]=\left\{\gamma \circ \tilde{f} \circ \gamma^{-1} \mid \gamma \in \mathfrak{D}\left(X^{\prime}\right)\right\}
$$

Teorema 1.7 Se $X$ '́ um poliedro compacto. $p: \widetilde{X} \rightarrow X$ seu recobrimento unicersal então: 
(i) $\operatorname{fir}(f)=\bigcup_{\tilde{f}} p(F i \cdot r(\tilde{f}))$ :

(ii) $[\tilde{f}]=\left[\tilde{f^{\prime}}\right] \Rightarrow p(F i x(\tilde{f}))=p\left(F i x\left(\tilde{f^{\prime}}\right)\right)$;

(iii) $[\tilde{f}] \neq\left[\tilde{f}^{\prime}\right] \Rightarrow p(F i x(\tilde{f})) \cap p\left(F i x\left(\tilde{f^{\prime}}\right)\right)=\emptyset$.

Demonstração: [Kïiang, pg 31].

Definição 1.8 O conjunto $p(F i x(\tilde{f})) \subset F i x(f)$, que pelo teorema 1.7 é determinado pela classe $[\tilde{f}]$, é denominado uma classe de ponto fixo de $f$.

Por simplicidade de notação denotaremos a classe $p(F i x(\tilde{f}))$ por $p(F i x([\tilde{f}])$.

Teorema 1.9 Se $X$ é um poliedro compacto $\epsilon f: X \rightarrow X$ é uma função contínua então

$$
F i x(f)=\bigcup_{[\tilde{f}]} p(F i x(\tilde{f}))
$$

onde $[\widetilde{f}]$ percorre as classes de levantamento da $f \in$ a união é disjunta.

Em outras palavras o conjunto de pontos fixos da $f$ se "decompoe" em uma união disjunta de classes de pontos fixos.

Demonstração: [Kiang, pg 31].

O teorema a seguir nos dá uma equivalência geométrica para a definição de classe de ponto fixo.

Teorema 1.10 Dois pontos $x_{0}, x_{1} \in F i x(f)$ estão na mesma classe de ponto fixo de $f: X \rightarrow X$ se, e somente se, existe uma curva $\zeta$ de $x_{0}$ até $x_{1}$ em $X$ tal que $\zeta \simeq f \circ \zeta \operatorname{rel}\{0,1\}$.

Demonstração: [Kïiang, pg 33] ou [Jiang, pg 6].

Teorema 1.11 Se $\mathbb{F}$ é uma classe de ponto fixo de $f: X \rightarrow X$, $X$ um poliedro compacto, então $\mathbb{F}$ é aberto $\epsilon$ m Firr $(f)$.

Demonstração: [Kiang. pg :35]. 
Corolário 1.12 f tem somente um número finito de classes de ponto firo nào cazias sendo cada uma delas um compacto $\mathrm{\epsilon m} X$.

Demonstração: [K̈̈iang, pg 3:]].

Vamos agora analisar a relaçào entre as classes de ponto fixo de $f$ e de $f^{\prime}$ quando $f \simeq f^{\prime}$.

Definição 1.13 Dada uma homotopia $H: X \times I \rightarrow X$ um levantamento de homotopia de $H$ é uma homotopia $\widetilde{H}: \widetilde{X^{\prime}} \times I \rightarrow \widetilde{X}$ tal que:

$$
H \circ(p \times i d)=\tilde{H} \circ p .
$$

O resultado a seguir é clássico da teoria de recobrimento.

Teorema 1.14 Dadas $H: X \times I \rightarrow X$ homotopia entre $f$ e ${\underset{\sim}{f}}^{\prime} ;$; $\tilde{f}$ um levantamento de $f$ então existe uma única $\widetilde{H}_{\tilde{f}}$, levantamento de $H$ tal que $\widetilde{H}_{\tilde{f}}(x, 0)=\widetilde{f}(x) \forall x \in \widetilde{X}$.

Usando esse teorema podemos estabelecer uma correspondência biunívoca entre os levantamentos de $f$ e os de $f^{\prime}$ tomando-se $\widetilde{f}^{\prime}(x)=\widetilde{H}_{\tilde{f}}(x, 1)$. Neste caso dizemos que $\tilde{f}$ e $\tilde{f}^{\prime}$ estão relacionados por $H$.

Observando que $\gamma \circ \widetilde{H}_{\tilde{f}}(x, t) \circ \gamma^{-1}$ é uma homotopia entre $\gamma \circ \tilde{f} \circ \gamma^{-1}$ e $\gamma \circ \tilde{f}^{\prime} \circ \gamma^{-1}$ temos que a correspondência também se realiza entre as classes de ponto fixo da $f$ e da $f^{\prime}$.

Definição 1.15 Se $H: X \times I \rightarrow X$ é uma homotopia a Representação Cilíndrica de $H$ é a aplicação $\mathfrak{H}: \mathrm{X} \times I \rightarrow X \times I$ dada por

$$
\mathfrak{H}(x, t)=(H(x, t), t) .
$$

Utm nível $H\left(x, t_{0}\right)$ de $H$ é chamado $t_{0}$-fatia de $\mathfrak{H}$.

Sabemos que se $p: \widetilde{X^{\prime}} \rightarrow X$ é o recobrimento universal de $X$ então $p \times i d: \widetilde{X^{\prime}} \times I \rightarrow$ $X \times I$ é o recobrimento universal de $X \times I$ e assim temos:

Lema 1.16 Se $H: X \times I \rightarrow X \in G: \tilde{X} \times I \rightarrow \widetilde{X}$ sào homotopias então $G \underset{e}{e}$ um levantamento de $H$ se. $\epsilon$ somente se, a representaşa cilíndrica $\mathfrak{G}: \widetilde{X} \times I \rightarrow \widetilde{X} \times I$ de $G$ '́ um levantamento da representação cilíndrica $\mathfrak{H}$ de $H$. 
Demonstração: [Kiang, pg 38].

Teorema 1.17 Dada $H: \mathrm{X} \times I \rightarrow X$ homotopia entre $f \in f^{\prime}$, se $\mathbb{F} \in \mathbb{F}^{\prime}$ sào classes de ponto firo de $f$ e de $f^{\prime}$ respectivamente então $\mathbb{F}$ está relacionada a $\mathbb{F}^{\prime}$ por $H$ se. $\epsilon$ somente se. $\mathbb{F} \in \mathbb{F}^{2}$ sào a 0-fatia $\epsilon$ a 1 -fatia de uma classe de ponto firo $(p \times$ $i d)(F i x(\tilde{\mathfrak{H}}))$ da representação cilíndrica $\mathfrak{H}$ de $H$.

Demonstração: [Kiang, pg 40].

Retomando o teorema 1.11 e o corolário 1.12 vemos que se $\mathbb{F}$ é uma classe de ponto fixo de $f$ o índice da $f$ em $\mathbb{F}$, ind $(f, \mathbb{F})$, está bem definido.

Definição 1.18 Uma classe de ponto fixo $\mathbb{F}$ de $f: X \rightarrow X^{\prime}$ é dita Classe Essencial se ind $(f, \mathbb{F}) \neq 0$; definimos ainda o Número de Nielsen de $f$, notaçào $N(f)$, como o número de classes essenciais da $f$.

Claramente $N(f)$ é um inteiro não negativo e, pelo corolário 1.12 , é finito.

Teorema $1.19 X$ poliedro compacto, $f: X \rightarrow X$ contínua então $N(f) \leq \# F i x(f)$.

Demonstração: Decorre facilmente da definição de $N(f)$ e das propriedades do índice.

Teorema 1.20 Se X é um poliedro compacto e $H: X \times I \rightarrow X^{\prime}$ é uma homotopia entre $f \in f^{\prime} ; \mathbb{F}$ e $\mathbb{F}^{\prime}$ são classes de ponto fixo de $f$ e de $f^{\prime}$ respectivamente e se $\mathbb{F}$ está relacionada com $\mathbb{F}^{\prime}$ por $H$ então:

$$
\operatorname{ind}(f, \mathbb{F})=\operatorname{ind}\left(f^{\prime}, \mathbb{F}^{\prime}\right) .
$$

Demonstração: [Kïiang, pg 47] ou [Jiang, pg 18].

O teorema acima pode ser entendido simplesmente como a afirmaçào de que a bijeção entre as classes de ponto fixo de $f$ e $f^{\prime}$ induzida por $H$ preserva índice.

Corolário $1.21 N(f)$ é um invariante homotópico e um limitante inferior para a cardinalidade do conjunto de pontos fixos de qualquer aplicaçào na classe de homotopia de $f$. 


\section{3 Índice de Coincidências}

Definição 1.22 L'ma coincidência entre duas funçòes $f_{1}, f_{2}: X \rightarrow Y$ é um ponto $x \in \mathrm{X}$ tal que $f_{1}(x)=f_{2}(x)$.

Chamaremos de $\operatorname{Coin}\left(f_{1}, f_{2}\right)$ o conjunto das coincidências do par $f_{1}, f_{2}$.

Suponhamos $f_{1}, f_{2}$ aplicaçòes entre $\mathrm{X}$ e $Y$ variedades compactas, $n$-dimensionais. conexas, orientáveis e sem bordo. Dado um aberto $W$ de $X$, se $K^{\circ}=C^{\prime} \operatorname{oin}\left(f_{1}, f_{2}\right) \cap W^{\circ}$ é compacto podemos definir um índice de coincidência do par $f_{1}, f_{2}$ em $W$ como a imagem da classe fundamental $\sim_{1}$ de $X^{\prime}$ pela composição:

$$
H_{n}(X) \rightarrow H_{n}(X, X \backslash V) \stackrel{\text { excisão }}{\longrightarrow} H_{n}(W . W \backslash V) \stackrel{\left(f_{1}, f_{2}\right)_{*}}{\longrightarrow} H_{n}(Y \times Y, Y \times Y \backslash \triangle(Y)) \approx \mathbb{Z}
$$

Onde $V$ é um aberto de $X$ com $K \subseteq V \subseteq \bar{V} \subseteq W$ ( $V$ existe pois $W$ é normal): $\left(f_{1}, f_{2}\right): W \rightarrow Y \times Y$ é dada por $\left(f_{1}, f_{2}\right)(x)=\left(f_{1}(x), f_{2}(x)\right)$ e, sendo $U_{2}$ a classe de Thom de $Y$, a identificação $H_{n}(Y \times Y, Y \times Y \backslash \triangle(Y)) \approx \mathbb{Z}$ é feita levando-se uma classe $\alpha$ no inteiro $\left\langle U_{2}, \alpha\right\rangle$.

Esse índice será denotado $I_{f_{1}, f_{2}}^{W}$ ou $\operatorname{ind}\left(f_{1}, f_{2}, K\right)$.

Observando que para $V^{\prime}$ tal que $K^{\prime} \subseteq V^{\prime} \subseteq \overline{V^{\prime}} \subseteq W$ o diagrama:

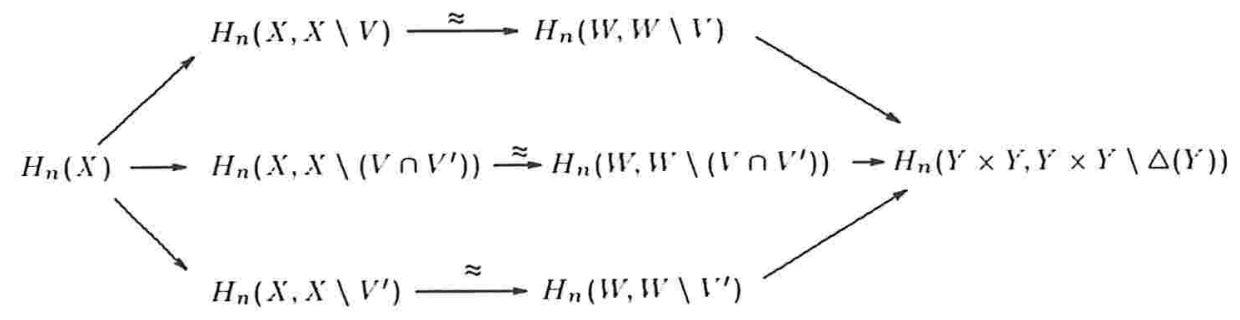

comuta temos que $I_{f_{1}, f_{2}}^{W}$ independe da escolha de $V$.

O índice acima definido goza das seguintes propriedades:

\section{Localização}

Se $W^{\prime}$ é outro aberto de $X^{\prime}$ e $g_{1}, g_{2}: W^{\prime} \rightarrow Y$ sào tais que $g_{1}=f_{1}$ e $g_{2}=f_{2} \mathrm{em}$ $W \cap W^{\prime \prime}$ e $\operatorname{Coin}\left(f_{1}, f_{2}\right) \cap W^{\prime}=K$ temos que:

$$
I_{f_{1}, f_{2}}^{W}=I_{g_{1}, g_{2}}^{W^{\prime}} .
$$

Demonstração: Decorre facilmente do diagrama acima. 


\section{Aditividade}

Sejam $f_{1} \cdot f_{2}: W \rightarrow Y$ com as propriedades acima e suponhamos $W=\bigcup_{i=1}^{k} \Psi_{i}$ $\operatorname{com} W_{i} \cap W_{j}=\emptyset$ se $i \neq j$ e que para todo $i, W_{i}$ é aberto de $\mathrm{X}$.

Entào:

$$
I_{f_{1}, f_{2}}^{W}=\sum_{i=1}^{k} I_{f_{1}, f_{2}}^{W_{1}}
$$

Demonstração: Em cada $W_{i}$ tomamos $V_{i}$ aberto de modo que $K \cap W_{i} \subseteq V_{i} \subseteq$ $\overline{V_{i}} \subseteq W_{i}$ : dessa forma $V_{i} \cap V_{j}=\emptyset$ se $i \neq j$. Definindo $V=\bigcup_{i=1}^{k} V_{i}$ basta olharmos para o seguinte diagrama comutativo:

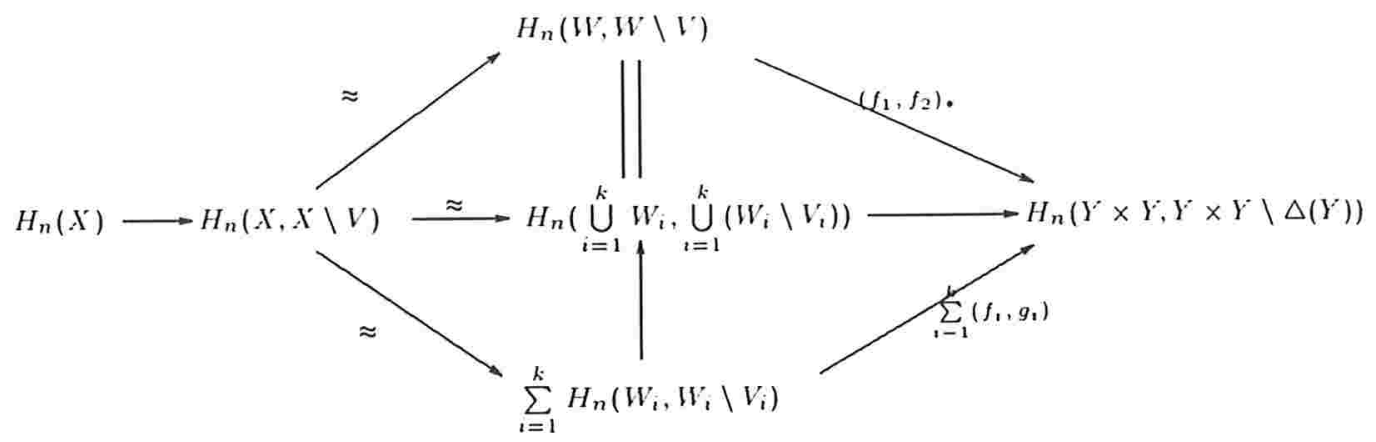

\section{Invariância homotópica}

Sejam $H_{1}(x . t)$ e $H_{2}(x, t)$ homotopias entre $\left(f_{1}, f_{2}\right)$ e $\left(f_{1}^{\prime}, f_{2}^{\prime}\right)$, denotando por $K_{t}$ o conjunto $\operatorname{Coin}\left(H_{1}(x, t), H_{2}(x, t)\right)$ para um $t$ fixado. Se $\bigcup_{t} K_{t}$ é compacto em $W$ entào:

$$
I_{f_{1}, f_{2}}^{W}=I_{f_{1}^{\prime}, f_{2}^{\prime}}^{W}
$$

Demonstração: Se $V$ é um aberto tal que $\bigcup_{t} K_{t} \subseteq V \subseteq \bar{V} \subseteq W$ as aplicaçōes:

$$
\left(H_{1}(x, t), H_{2}(x, t)\right):(W, W \backslash V) \rightarrow(Y \times Y, Y \times Y \backslash \triangle(Y))
$$

induzem o mesmo homomorfismo de grupos para todo $t$. Assim:

$$
\left(f_{1}, f_{2}\right)_{\star}=\left(f_{1}^{\prime}, f_{2}^{\prime}\right)_{\star} \text { e } I_{f_{1}, f_{2}}^{W}=I_{f_{1}^{\prime}, f_{2}^{\prime}}^{W}
$$




\section{Multiplicatividade}

Sejam $f_{1}, f_{2}$ aplicaçòes entre $X_{1}$ e $Y_{1}$, variedades n-dimensionais. conexas. orientáveis e $g_{1}, g_{2}$ aplicaçòes entre $X_{2}$ e $Y_{2}$ variedades $m$-dimensionais também orientáveis e conexas. Se $W$ e $W^{\prime \prime}$ sào abertos $\operatorname{com} C \operatorname{oin}\left(f_{1}, f_{2}\right) \cap W$ e $C \operatorname{oin}\left(g_{1}, g_{2}\right) \cap$ $W^{\prime}$ compactos entào:

$$
I_{f_{1} \times g_{1}, f_{2} \times g_{2}}^{W \times W^{\prime}}=\left(I_{f_{1}, f_{2}}^{W}\right) \cdot\left(I_{g_{1}, g_{2}}^{W}\right) .
$$

Demonstração: Sendo $z_{1}$ e $z_{2}$ as classes fundamentais de $H_{n}\left(X_{1} ; \mathbb{Q}\right)$ e $H_{m}\left(X_{2} ; \mathbb{Q}\right)$ respectivamente. Sabemos que $z_{1} \times z_{2}$ é a classe fundamental de $X_{1} \times X_{2}$. Assim temos

$$
\begin{aligned}
I_{f_{1} \times f_{2}}^{X_{1} \times X_{2}} & =\left(f_{1} \times f_{2}\right)_{\times}\left(z_{1} \times z_{2}\right) \\
& =\left[f_{1 *}\left(z_{1}\right)\right] \times\left[f_{2 *}\left(z_{2}\right)\right] \\
& =\left(I_{f_{1}, f_{2}}^{W}\right) \cdot\left(I_{g_{1}, g_{2}}^{W^{\prime}}\right) .
\end{aligned}
$$

É bastante interessante notar que:

Proposição 1.23 Dadas $f_{1}, f_{2}$ aplicações como as definidas acima. Se Coin $\left(f_{1}, f_{2}\right) \cap$ $W=\emptyset$ temos $I_{f_{1}, f_{2}}^{W}=0$.

Demonstração: Se $f_{1}$ e $f_{2}$ nào têm coincidência alguma em $W$ dado um aberto $V$ nas condições requeridas para o cálculo do índice temos que a aplicação

$$
\left(f_{1}, f_{2}\right):(W, W \backslash V) \rightarrow(Y \times Y, Y \times Y \backslash \triangle(Y))
$$

pode ser escrita na forma

$$
\left(f_{1}, f_{2}\right):(W . W \backslash V) \rightarrow(Y \times Y \backslash \triangle(Y), Y \times Y \backslash \triangle(Y)),
$$

o que deixa claro que $\left(f_{1}, f_{2}\right)_{\times}$é o homomorfismo nulo.

Corolário 1.24 Se $I_{f_{1}, f_{2}}^{W} \neq 0$ então $f_{1} \in f_{2}$ têm pelo memos uma coincidência $\epsilon m$ $W$. 
Vamos agora dar uma interpretaçào geométrica para o índice de uma concidência isolada.

Se definirmos $F: D^{n} \times D^{n} \rightarrow D^{n}$ por:

$$
F(x, y)=\frac{1}{2}(y-x)
$$

Restringindo $F$ temos:

$$
F:\left(D^{n} \times D^{n}, D^{n} \times D^{n} \backslash \triangle\left(D^{n}\right)\right) \rightarrow\left(D^{n}, D^{n} \backslash 0\right) .
$$

Tomando $j: D^{n} \rightarrow D^{n} \times D^{n}, j(w)=(0, w)$ e fazendo $H(x . t): D^{n} \times I \rightarrow D^{n} \mathrm{e}$ $G(x, t): D^{n} \times D^{n} \times I \rightarrow D^{n} \times D^{n}$ definidas por

$$
H(x, t)=\frac{x}{1+t} \quad \text { e } \quad G(x, y, t)=\left(\frac{(1-t) x}{1+t}, \frac{y-t x}{1+t}\right)
$$

temos que:

$$
H(x, 0)=x, H(x, 1)=F \circ j(x) \text { e } H(x, t) \in D^{n} \backslash 0 \forall x \neq 0,0 \leq t \leq 1
$$

e

$$
\begin{gathered}
G(x, y, 0)=(x, y), G(x, y, 1)=j \circ F(x, y) \\
\operatorname{com} \\
G(x, y, t) \in D^{n} \times D^{n} \backslash \triangle\left(D^{n}\right) \quad \forall(x, y), x \neq y, 0 \leq t \leq 1 .
\end{gathered}
$$

Assim $F$ é uma equivalência homotópica, sendo $j$ sua inversa homotópica.

Considere $W$ um aberto de $\mathrm{X}^{\prime}$ e a uma coincidência isolada de $\left(f_{1}, f_{2}\right)$ com $\operatorname{Coin}\left(f_{1}, f_{2}\right) \cap W=\{a\}$. Como $X$ e $Y$ são $n$-variedades, existem fechados $U \subset Y$ e $\bar{V} \subset X(V$ aberto em $X)$ homeomorfos, por $h,(h(a)=0)$ e $k$ respectivamente, a $D^{n}$ de modo que $\bar{V} \subseteq W \cap f_{1}^{-1}(U) \cap f_{2}^{-1}(U)$. Assumindo que $h$ e $k$ preservam orientações definimos $\phi: S^{n-1} \rightarrow S^{n-1}$ pela composiçào:

$$
S^{n-1} \stackrel{k^{-1}}{\longrightarrow} \partial \bar{V} \stackrel{\left(f_{1}, f_{2}\right)}{\longrightarrow} Y \times Y \backslash \triangle(Y) \stackrel{h \times h}{\longrightarrow} D^{n} \times D^{n} \backslash \triangle\left(D^{n}\right) \stackrel{F}{\longrightarrow} D^{n} \backslash 0 \stackrel{\pi}{\longrightarrow} S^{n-1}
$$

onde $\pi$ é a projeção central.

Proposição 1.25 Dadas $f_{1}, f_{2}$ nas condiçòes acima, se W' ú um aberto de $\mathrm{X}$ com C oin $\left(f_{1}, f_{2}\right) \cap W=\{a\}$ então:

$$
I_{f_{1}, f_{2}}^{\mathrm{II}}=\operatorname{deg}(0)
$$


Demonstração: Escolhemos a gerador de $H_{n-1}\left(\varsigma^{n-1}\right)$ como a imagem de $z_{1}$ (classe fundamental de $X$ ) pela composiçào:

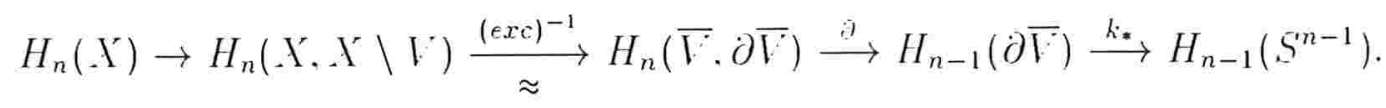

Dessa forma. denotando por $i$ a inclusào, temos o seguinte diagrama comutativo:

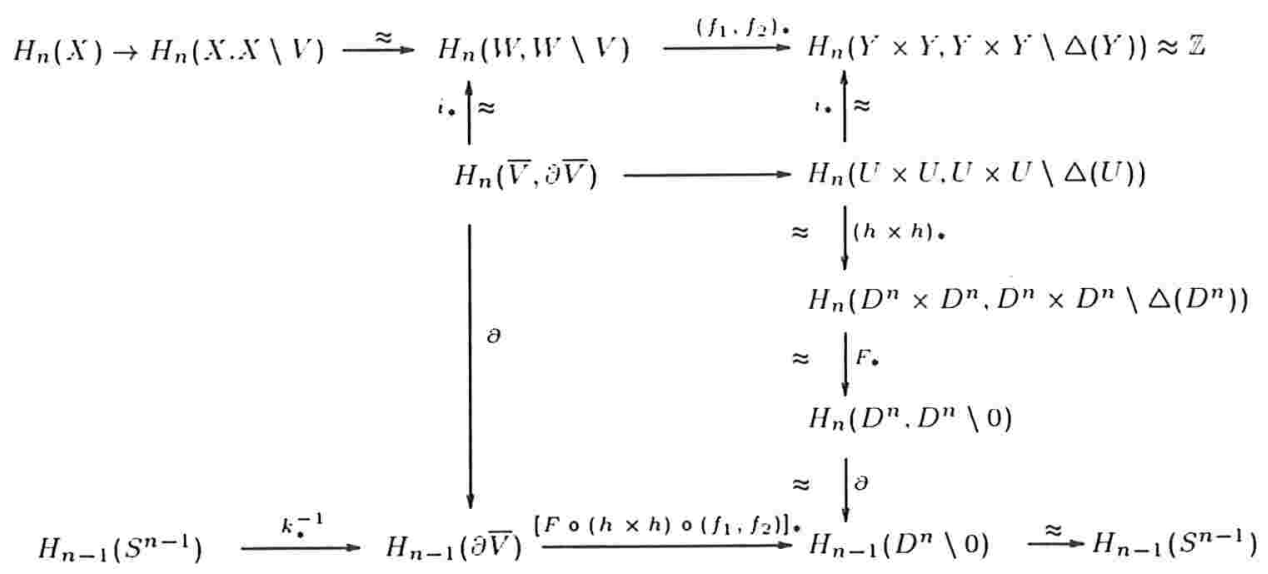

Observando que o gerador de $H_{n}(Y \times Y, Y \times Y \backslash \triangle(Y))$ é levado no gerador escolhido de $H_{n-1}\left(S^{n-1}\right)$ verificamos que o inteiro associado à imagem de $z_{1}$ na parte superior do diagrama corresponde ao grau da $\phi$ (parte de baixo).

De maneira mais simples podemos dizer que, nas condições de 1.25

$$
I_{f_{1}, f_{2}}^{W}=\operatorname{deg}\left(\left.\left(f_{2}-f_{1}\right)\right|_{i \bar{V}}\right) .
$$

\subsection{Número de Lefschetz}

Um dos primeiros resultados obtido na teoria de ponto fixo utilizando invariantes homológicos foi o que chamamos hoje Número de Lefschetz $L(f)$ de uma aplicaçào $f$ que é definido como:

$$
L(f)=\sum_{k=0}^{\infty}(-1)^{k} \operatorname{tr}\left(f_{k}\right) .
$$

onde $f: M \rightarrow M, M$ variedade fechada, $f_{k}: H_{k}(M, \mathbb{Q}) \rightarrow H_{k}(M, \mathbb{Q})$ é o homomorfismo induzido pela $f$ nos grupos de homologia de $M$ com coeficientes racionais.

Como $f_{k}$ é invariante homotópico. $L(f)$ está definido nas classes de homotopia das aplicações de $M$ em $M$. Se $M$ é uma variedade fechada valem: 


\section{Existência}

Se $L(f) \neq 0$ entào $f$ tem pelo menos um ponto fixo.

\section{Normalização}

Sejam . I uma variedade fechada orientada e $f:$ I $\rightarrow$ M uma aplicação. tal que Fir $(f)=\bigcup_{k=1}^{r} F_{k}$ com $F_{i}$ aberto e fechado em $F i x(F)$ para todo $i$. Se $\left\{a_{1}, a_{2}, \ldots, a_{r}\right\}$ é um conjunto de inteiros tal que ind $\left(f, F_{i}\right)=a_{i}$ então:

$$
L(f)=\sum_{i=i}^{r} a_{i} .
$$

Também decorre facilmente da definição que se $f \simeq i d$ temos:

$$
L(f)=\backslash(M)
$$

onde $\backslash(M)$ é a característica de Euler da variedade.

É preciso ressaltar que a teoria de ponto fixo não se limita à variedades; podemos definir, de maneira análoga, número de Lefschetz e índice para $f:\left|K^{\prime}\right| \rightarrow|K| \operatorname{com}\left|K^{\prime}\right|$ a realização geométrica de um complexo simplicial ou de maneira mais geral ainda $|K|$ um ENR (retrato de vizinhança euclidiana) de modo que as duas propriedades acima continuem válidas.

A generalizaçào para coincidências do número de Lefschetz decorre do diagrama:

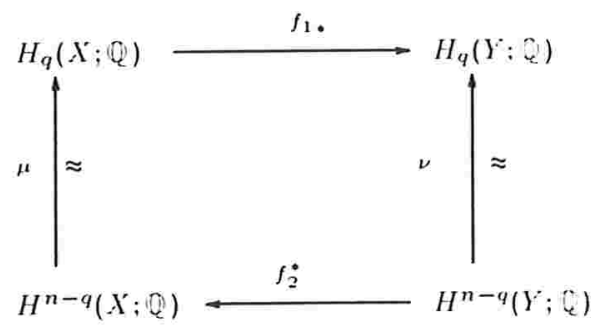

Onde $\mu$ e $\nu$ sào os isomorfismos dados pela dualidade de Poincaré.

Tomamos $\theta_{q}: H_{q}(X ; \mathbb{Q}) \rightarrow H_{q}(X ; \mathbb{Q})$ como:

$$
\theta_{q}(\alpha)=\mu \circ f_{2}^{*} \circ \nu^{-1} \circ f_{1_{x}}(\alpha) \text {. }
$$


Definição 1.26 Dadas $f_{1} . f_{2}: X \rightarrow Y$ aplicaşòes com $X \in Y$ n-cariedades fechadas e orientadas o Número de Lefschetz. $L\left(f_{1}, f_{2}\right)$. $\epsilon$ dado por:

$$
L\left(f_{1}, f_{2}\right)=\sum_{q=0}^{n}(-1)^{q} \operatorname{tr}\left(\theta_{q}\right) .
$$

Proposição 1.27 Se definirmos $\theta_{n-q}^{\prime}: H^{n-q}(X ; \mathbb{Q}) \rightarrow H^{n-q}\left(X^{\prime}: \mathbb{Q}\right)$ fazendo $\theta_{n-q}^{\prime}(\alpha)=$ $\nu^{-1} \circ f_{1 *} \circ \mu \circ f_{2}^{\times}(\alpha)$, teremos:

$$
\sum_{q=0}^{n}(-1)^{q} \operatorname{tr}\left(\theta_{q}^{\prime}\right)=(-1)^{n} L\left(f_{1}, f_{2}\right)
$$

Demonstração: [Vick, pg 184].

Teorema 1.28 Dadas $f_{1}, f_{2}: X \rightarrow Y$ com $X$ e $Y$ variedades n-dimensionais, fechadas, conexas e orientáveis. então:

$$
I_{f_{1}, f_{2}}^{X}=L\left(f_{1}, f_{2}\right) .
$$

Demonstração: [Vick, pg 187].

Corolário $1.29 L\left(f_{1}, f_{2}\right) \neq 0 \Rightarrow \operatorname{Coin}\left(f_{1}, f_{2}\right) \neq \emptyset$.

Definição 1.30 Seja $f: X \rightarrow Y$ uma aplicaçào entre cariedades n-dimensionais. orientáveis e compactas: o Grau de $f$, $\operatorname{deg}(f)$, é o inteiro tal que $f_{\times}\left(z_{1}\right)=\operatorname{deg}(f) \cdot z_{1}^{\prime}$, onde $z_{1} \in H_{n}(X ; \mathbb{Q})$ e $z_{1}^{\prime} \in H_{n}(Y ; \mathbb{Q})$ são as classes fundamentais de $X^{\prime}$ e $Y$.

Teorema 1.31 Sejam $f, X$ X $Y$ como na definição acima. Se $f^{\prime} \simeq f$ então:

$$
I_{f, f^{\prime}}^{X}=(-1)^{n} \cdot \operatorname{deg}(f) \cdot \backslash(Y) .
$$

Demonstração: Tomamos $\alpha_{1}, \ldots, \alpha_{r}$ base de $H_{q}\left(X^{r}: \mathbb{Q}\right), \beta_{1}, \ldots, \beta_{k}$ base de $H_{q}(Y ; \mathbb{Q})$, $\alpha_{1}^{\prime}, \ldots, \alpha_{r}^{\prime}$ base de $H^{q}(X ; \mathbb{Q})$ e $\beta_{1}^{\prime}, \ldots, \beta_{k}^{\prime}$ base de $H^{q}(Y ; \mathbb{Q})$ tais que $\mu\left(\alpha_{i}^{\prime}\right)=\alpha_{i}$ e $\nu\left(\beta_{i}^{\prime}\right)=\beta_{i} \quad \forall i$. Assim:

$$
\theta_{q}^{\prime}\left(\beta_{i}^{\prime}\right)=\nu^{-1} \circ f_{\times} \circ \mu \circ f^{*}\left(3_{i}^{\prime}\right)
$$

Entào 


$$
\nu \circ \theta_{\eta}^{\prime}\left(\beta_{i}^{\prime}\right)=f_{\times} \circ \mu \circ f^{*}\left(\beta_{i}^{\prime}\right)=f_{\times}\left(f^{*}\left(\beta_{i}^{\prime}\right) \cap z_{1}\right)
$$

e. pela naturalidade do produto cap.

$$
f_{\star}\left(f^{\times}\left(\beta_{i}^{\prime}\right) \cap z_{1}\right)=\beta_{i}^{\prime} \cap f_{\times}\left(z_{1}\right)=\operatorname{deg}(f) \cdot\left(\beta_{i}^{\prime} \cap z_{1}^{\prime}\right)=\operatorname{deg}(f) \cdot 3_{i} .
$$

Assim

$$
\theta_{q}^{\prime}\left(\beta_{i}^{\prime}\right)=\operatorname{deg}(f) \cdot \nu^{-1}\left(\beta_{i}\right)=\operatorname{deg}(f) \cdot \beta_{i}^{\prime} .
$$

Dessa forma $\operatorname{tr}\left(\theta_{q}^{\prime}\right)=\operatorname{deg}(f) \cdot k=\operatorname{deg}(f) \cdot \operatorname{dim} H_{q}(Y ; \mathbb{Q})$.

De onde concluímos que:

$$
\sum_{q=0}^{n} \operatorname{tr}\left(\theta_{q}^{\prime}\right)=\operatorname{deg}(f) \cdot \checkmark(Y) .
$$

Pelo corolário 1.27 e pelo teorema 1.31 temos o desejado.

\subsection{Número de Nielsen para Coincidência}

Apesar de só ser conhecida uma definição de índice de coincidência para aplicações entre variedades podemos extender a idéia de classes para coincidências de aplicações entre espaços mais gerais.

Se $X$ e $Y$ sào poliedros conexos, $X$ compacto, $p: \tilde{X} \rightarrow X$ e $q: \tilde{Y} \rightarrow Y$ seus recobrimentos universais valem as seguintes proposições:

Proposição 1.32 Se $\tilde{f}: \tilde{X} \rightarrow \widetilde{Y}$ é um levantamento fixado de $f$ existe $\tilde{f}_{\pi}: \mathfrak{D}\left(X^{\prime}\right) \rightarrow$ $\mathfrak{D}\left(Y^{*}\right)$ endomorfismo tal que:

$$
\tilde{f}_{\pi}(\gamma) \circ \tilde{f}=\tilde{f} \circ \gamma
$$

Demonstração: [Jiang, pg 25].

Proposição 1.33 Se $\tilde{f}_{1}$ e $\tilde{f}_{1}^{\prime}$ são levantamentos de $f_{1}: X \rightarrow Y$ e $\widetilde{f}_{2}$ e $\widetilde{f}_{2}^{\prime}$ são levantamentos de $f_{2}: X^{\prime} \rightarrow Y$ então $p\left(\operatorname{Coin}\left(\tilde{f}_{1}, \widetilde{f}_{2}\right)\right)=p\left(C^{\prime} \operatorname{oin}\left(\tilde{f}_{1}^{\prime}, \widetilde{f}_{2}^{\prime}\right)\right)$ se, e somente se. existirem $\gamma \in \mathfrak{D}(X)$ e $\alpha \in \mathfrak{D}(Y)$ tais que:

$$
\tilde{f}_{1}^{\prime}=\alpha \circ \tilde{f}_{1} \in \tilde{f}_{2}^{\prime}=\alpha \circ \tilde{f}_{2 \pi}(\gamma) \circ \tilde{f}_{2} \circ \gamma^{-1} \text {. }
$$


Demonstração: [Oliveira. pg 2:5].

Repetindo os passos dados para ponto fixo temos:

Definição 1.34 Sejam $\widetilde{f}_{1} \in \widetilde{f}_{1}^{\prime}$ levantamentos de $f_{1} ; \widetilde{f}_{2}$ e $\tilde{f}_{2}^{\prime}$ levantamentos de $f_{2}$. $O \operatorname{par}\left(\widetilde{f}_{1}, \widetilde{f}_{2}\right)$ é equivalente à $\left(\widetilde{f}_{1}^{\prime}, \widetilde{f}_{2}^{\prime}\right)$ se existem $; \in \mathfrak{D}\left(X^{\prime}\right)$ e a $\in \mathfrak{D}(Y)$ tais que:

$$
\tilde{f}_{1}^{\prime}=\alpha \circ \tilde{f}_{1} \in \tilde{f}_{2}^{\prime}=\alpha \circ \tilde{f}_{2 \pi}(\gamma) \circ \tilde{f}_{2} \circ \gamma^{-1} \text {. }
$$

As classes de equivalência assim determinadas são chamadas Classes de Levantamento de $f_{1}$ e $f_{2}$.

Usando a proposição 1.33 podemos passar a noção de classe para as coincidências de $f_{1}$ e $f_{2}$.

Definição $1.35 O$ cojunto $p\left(\operatorname{Coin}\left(\tilde{f}_{1}, \widetilde{f}_{2}\right)\right) \subset \operatorname{Coin}\left(f_{1}, f_{2}\right)$, determinado pela classe $\left[\left(\widetilde{f}_{1}, \widetilde{f}_{2}\right)\right]$ é denominado Classe de Coincidência de $f_{1}$ e $f_{2}$.

Corolário 1.36 Coin $\left(f_{1}, f_{2}\right)$ é a união disjunta de classes de coincidência.

Analogamente ao caso de ponto fixo temos:

Teorema 1.37 Dois pontos $x_{0}, x_{1} \in \operatorname{Coin}\left(f_{1}, f_{2}\right)$ estão na mesma classe de coincidência de $f_{1}, f_{2}: X \rightarrow Y$ se, e somente se. existe uma curva $\zeta$ de $x_{0}$ até $x_{1}$ em $X$ tal que $f_{1} \circ \zeta \simeq f_{2} \circ \zeta \operatorname{rel}\{0,1\}$.

Demonstração: [Oliveira. pg :30].

Corolário 1.38 Se $\mathbb{F} \subset$ Coin $\left(f_{1}, f_{2}\right)$ é uma classe de coincidência então $\mathbb{F}$ é um aberto $\mathrm{em} \mathrm{C}^{\prime} \operatorname{oin}\left(f_{1}, f_{2}\right)$

Demonstração: [Oliveira. pg 30].

Corolário 1.39 Coin $\left(f_{1}, f_{2}\right)$ contém somente um múmero finito de classes não cazias.

Demonstração: [Oliveira. pg 30]. 
Corolário 1.40 Se $\mathbb{F}$ é uma classe de coincidencia de $f_{1}, f_{2}$ então $\mathbb{F}$ é um compacto $\epsilon m$ X.

Demonstração: [Oliveira. pg :31].

Teorema 1.41 Sejam $f_{1}, f_{1}^{\prime}, f_{2}, f_{2}^{\prime}: X^{\prime} \rightarrow Y$. $X \in Y$ poliedros coneros, $X$ compacto. $f_{1} \simeq f_{1}^{\prime} \in f_{2} \simeq f_{2}^{\prime}$ entào:

(i) As classes de coincidência de $f_{1}, f_{2}$ estão em correspondência biunívoca com as de $f_{1}^{\prime}, f_{2}^{\prime}$.

(ii) Se $X \in Y$ forem variedades n-dimensionais, conexas, sem bordo, orientadas $\epsilon$ compactas então a correspondência dada em (i) preserva índice.

Demonstração: (i) [Oliveira, pg 31].

(ii) [Oliveira. Apendice I pp 118:128].

É interessante ressaltar que a correspondência obtida nesse teorema é do mesmo tipo da usada no caso de ponto fixo, no sentido de que uma homotopia entre $\left(f_{1}, f_{2}\right)$ e $\left(f_{1}^{\prime}, f_{2}^{\prime}\right)$ leva cada classe de coincidência continuamente em classes de coincidência dos níveis intemediários.

Definição 1.42 Sejam $\left(f_{1}, f_{2}\right): X \rightarrow Y$ aplicações entre variedades $n$-dimensionais. conexas, orientadas, compactas e sem bordo. Uma classe de coincidência $\mathbb{F}$ é dita uma Classe Essencial se ind $\left(f_{1}, f_{2}, \mathbb{F}\right) \neq 0$. O número de classes essenciais de $f_{1}, f_{2}$ é o Número de Nielsen, $N\left(f_{1}, f_{2}\right)$, de $f_{1}, f_{2}$.

Novamente $V\left(f_{1}, f_{2}\right)$ é um inteiro, finito e não negativo.

Teorema 1.43 Sejam $f_{1}, f_{2}$ como na definição acima: entĩo:

$$
N\left(f_{1}, f_{2}\right) \leq \# C \operatorname{oin}\left(f_{1}, f_{2}\right) .
$$

Demonstração: Basta olhar para o corolário 1.24.

Teorema $1.44 \quad Y\left(f_{1}, f_{2}\right)$ é um invariante homotópico.

Demonstração: Aplicação imediata do teorema 1.41. 


\section{Capítulo 2}

\section{Localização de Pontos Fixos}

Nesse capítulo vamos apresentar alguns resultados disponíveis em [Schimer 1] e [Schimer 2] sobre a localização de pontos fixos em poliedros.

Inicialmente vamos estabelecer uma notação básica para alguns elementos usuais:

- se $K$ é um complexo simplicial denotaremos por $|K|$ sua realização geométrica; no caso de um simplexo $\sigma,|\sigma|$ será seu interior em $\left|\kappa^{\circ}\right|$;

- $\sigma(x)$ será o "carrier" do ponto $x$, ou seja o único simplexo que contém $x$ em seu interior;

- a estrela de um simplexo $\sigma$ (definida como a uniào do interior de todos os simplexos que contenham $\sigma$ como face) será indicada st $(\sigma)$;

- $[x, y]$ será o segmento de reta de $x$ até $y$;

- $\mathcal{V}(x)$ será a união de todos os $\left|\sigma_{i}\right|$ tais que $\overline{\left|\sigma_{i}\right|} \cap \sigma(x) \neq \emptyset$;

- para $A \subset|K|$, definimos

$$
U(A, \Xi)=\{x \in|K| \mid d(x, A)<\varepsilon\}
$$

com $d$ uma métrica em $|K|$.

\section{Lema 2.1 Definindo}

$$
N=\left\{(x, y) \in|K| \times\left|K^{\prime}\right| \mid \sigma(x) \cap \sigma(y) \neq \emptyset\right\} .
$$

Entrio existe uma aplicaçìo $h:|K| \times I \rightarrow|K|$ tal que: 
(i) $h(x, y, 0)=x: \quad h(x, y, 1)=y$

(ii) $h(x, x \cdot t)=x \quad \forall t \in I$

(iii) $h(x, y, t) \neq x$ se $x \neq y$ e $t \neq 0$.

Demonstração: [Brown. pg 124].

Definição 2.2 Uma aplicaçào $f: U \rightarrow\left|{ }^{*}\right|$ com $U \subseteq\left|K^{\prime}\right|$ é dita próxima se para todo $x \in U$ temos $f(x) \in \mathcal{V}(x)$.

Corolário 2.3 Se $f:\left|K^{\prime}\right| \rightarrow\left|K^{\prime}\right|$ é uma aplicação prórima então $f$ é uma deformaçào.

Demonstração: Usando o lema 2.1 temos que $f \simeq$ id.

Lema 2.4 Sejam $\sigma_{1}, \sigma_{2} \in K$ dois simplexos de dimensão maior que um tais que $\sigma_{1} \cap \sigma_{2}$ tem dimensaio maior que zero. Se $a \in\left|\sigma_{1}\right|, b \in\left|\sigma_{1} \cap \sigma_{2}\right|$ e se $f:\left|K^{\prime}\right| \rightarrow|K|$ é uma aplicação que satisfaz:

(1) a é um ponto fixo isolado de $f$; ind $(f, a) \neq 0$ e Fix $(f) \cap[a, b]=\{a\}$;

(Q) f é um aplicaçào próxima em $[a, b]$.

Então existe $\Xi>0$ e uma aplicação $f^{\prime}:\left|K^{\prime}\right| \rightarrow\left|K^{\prime}\right|$ tal que:

(i) $f^{\prime}$ é homotópica a $f$ e $f^{\prime}=f$ em $|K| \backslash U([a, b], \varepsilon)$ :

(ii) $F i x\left(f^{\prime}\right) \cap \bar{U}([a, b], \varepsilon)=c$ para algum $c \in U(b, \varepsilon) \cap\left|\sigma_{2}\right|$;

(iii) $f^{\prime}$ é próxima $\in \mathrm{m} \bar{U}([a, b], \varepsilon)$,

onde $\bar{L}([a, b], \Xi)$ é o fecho de $U([a, b], \Xi)$.

Demonstração: [Brown. pg 12S].

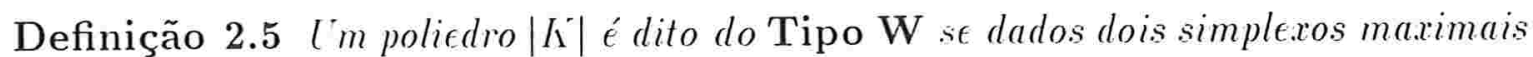
$\sigma, \sigma^{\prime} \in K^{\prime}$ existe uma sequência $\sigma=\sigma_{1}, \sigma_{2}, \ldots, \sigma_{r}=\sigma^{\prime}$ de simpleros maximais de $\mathrm{K}^{\circ}$ tal que $\sigma_{i} \cap \sigma_{i+1}$ tem dimensào maior que zero. $i=1 \ldots . . .-1$. 
Lema 2.6 Se $\mid$ Si é um poliedro do tipo W'entio triste uma aplicaçào prórima f : $\left|h^{\prime}\right| \rightarrow\left|K^{\prime}\right|$ tal que:

(i) $\backslash\left(\left|K^{\prime}\right|\right)=0 \Rightarrow F i x(f)=\emptyset$.

(ii) $\backslash(|K|) \neq 0 \Rightarrow F i x(f)=\{a\}$ com $\sigma(a)$ um simplexo maximal.

Demonstração: Na demonstração do Teorema 1 em [Brown, pg 143] constrói-se uma aplicaçào, lá denominada $f^{\prime}$, que satisfaz as condições exigiclas.

Lema 2.7 Sejam $\sigma_{1}, \sigma_{2}$ simplexos maximais de um poliedro $|K|$ do tipo $W$. Se $f$ : $\left|K^{\prime}\right| \rightarrow\left|K^{\prime}\right|$ é uma aplicação próxima com a $\in\left|\sigma_{1}\right|$ seu único ponto fixo. Então existe $f^{\prime}:\left|\Lambda^{\prime}\right| \rightarrow\left|\Lambda^{i}\right|$ uma aplicação próxima com fix $\left(f^{\prime}\right)=a^{\prime}$ com $a^{\prime} \in\left|\sigma_{2}\right|$.

Demonstração: Obtemos o resultado pela aplicação repetida do lema 2.4.

Lema 2.8 Seja $\left|K^{\prime}\right|$ um poliedro do tipo $W$ com $\chi\left(\left|K^{\prime}\right|\right) \neq 0$. Se $a \in\left|K^{\prime}\right| \dot{\epsilon}$ um ponto qualquer então existe uma aplicação próxima $f:\left|K^{\prime}\right| \rightarrow|K| \operatorname{com}$ Fix $(f)=\{a\}$.

Demonstração: Existe $\sigma$ um simplexo maximal de $K$ com $a \in \bar{\sigma}$. Usando os lemas 2.6 e 2.7 obtemos uma aplicação próxima $f_{1}:|K| \rightarrow|K|$ com exatamente um ponto fixo $b$ com $b \in|\sigma|$. Tomamos $\eta>0$ de modo que $\bar{U}([a, b], \eta) \subset|\operatorname{st}(\sigma(a))|$ usando o lema 2.1 obtemos um $t^{*}=t^{*}(x), 0<t^{*} \leq 1$, tal que para todo ponto $x \in \partial U\left([a, b], \frac{\eta}{2}\right)$ temos

$$
h\left(x, f_{1}(x), t\right) \subset|s t(\sigma(a))| \text { se } 0 \leq t \leq t^{*}(x) .
$$

Assim existe um $\varepsilon(x)>0$ tal que

$$
h\left(x^{\prime} \cdot f_{1}\left(x^{\prime}\right), t\right) \subset|s t(\sigma(a))| \quad \forall x^{\prime} \in \partial U\left([a, b], \frac{\eta}{2}\right) \cap U(x, \varepsilon(x)) \text { e } 0 \leq t \leq t^{\times}(x) .
$$

Criamos assim uma cobertura aberta do compacto $\partial U\left([a, b], \frac{\eta}{2}\right)$ a saber:

$$
\left\{U(x, \Xi(x)) \mid x \in \partial U\left([a, b], \frac{\eta}{2}\right)\right\} .
$$

Tomando

$$
\left\{U\left(x_{i}, \Xi\left(x_{i}\right)\right) \mid x_{i} \in \partial U\left([a, b], \frac{\eta}{2}\right), i=1, \ldots r r\right\}
$$

uma subcobertura finita e fazendo

$$
t_{0}=\min \left\{t^{*}\left(x_{1}\right), \ldots t^{*}\left(x_{r}\right)\right\}
$$


temos que, se $x \in \partial U\left([a, b], \frac{\eta}{2}\right)$ entào $h\left(x, f_{1}(x), t\right) \subset|s t(\sigma(x))|$ para todo $0 \leq t \leq t_{0}$.

Denotando por $x_{\frac{\eta}{2}}$ e $x_{\eta}$ os pontos de intersecçào da semi-reta $\vec{a} \cdot \vec{x} \operatorname{com} L\left([a, b] \cdot \frac{\eta}{2}\right)$ e $\partial U([a, b], \eta)$ respectivamente, definimos:

$$
f(x)= \begin{cases}f_{1}(x) & \text { se } x \in|K| \backslash \bar{U}([a, b], \eta) \\ h\left(x, f_{1}(x),\left(1-t_{0}\right) \lambda+t_{0}\right) & \text { se } x=(1-\lambda) x_{\frac{\eta}{2}}+\lambda x_{\eta} \text { e } 0 \leq \lambda \leq 1 \\ (1-\lambda) a+\lambda h\left(x_{\frac{\eta}{2}}, f_{1}\left(x \frac{\eta}{2}\right), t_{0}\right) & \text { se } x=(1-\lambda) a+\lambda x_{\frac{\eta}{2}} \text { e } 0 \leq \lambda \leq 1 \\ a & \text { se } x=a .\end{cases}
$$

$f$ satisfaz as condições desejadas.

Teorema 2.9 Seja $\left|K^{\prime}\right|$ um poliedro do tipo W. Se $A \subset\left|K^{\circ}\right|$ é um fechado, com $A \neq \emptyset$ se $\backslash(|K|) \neq 0$ então existe uma aplicação $f:|K| \rightarrow|K|$ com $F i x(f)=A$.

Demonstração: Tomamos $\delta$ o diâmetro de $\left|K^{\prime}\right|$ e fazemos $t(x)=\frac{d(x, A)}{\delta}$

Caso $1 \backslash\left(\left|K^{\prime}\right|\right)=0$

Se $A=\emptyset$ o lema 2.6 nos diz que existe $f:|K| \rightarrow\left|K^{\prime}\right|$ próxima livre de ponto fixo.

Se $A \neq \emptyset$ tomamos $g:|K| \rightarrow|K|$ próxima, livre de ponto fixo e definimos $f(x)=h(x, g(x), t(x))$, onde $h$ é dada pelo lema 2.1. Assim Fix $(f)=A$.

Caso $2 \gamma\left(\left|\kappa^{\prime}\right|\right) \neq 0$

Tomamos $a \in A$, pelo lema 2.8 , existe $g:|\Lambda| \rightarrow|\Lambda|$ próxima com Fix $(g)=$ $\{a\}$. Definimos então $f(x)=h(x, g(x) . t(x))$, novamente $h$ é dada pelo lema 2.1. Assim Fix $(f)=A$.

Definição 2.10 Dizemos que um espaço topológico X tem a Propriedade da Invariância Completa se qualquer $A \subset X$. fechado. pode ser realizado com conjunto de pontos fixos de uma aplicação $f: X \rightarrow X^{\prime}$.

Corolário 2.11 Se X' é uma variedade $n$-dimensional $(n \geq 2)$ triangularizável entào $\mathrm{X}$ tem a propriedade da invariância completa.

Demonstração: Claramente, nessas condiçòes $X$ é um poliedro do tipo $\mathrm{W}$.

Nosso objetivo agora consiste em conseguir resultados de localização para aplicaçòes que nào sào próximas. Para tanto vamos enunciar alguns lemas técnicos apresentados em [Brown]. 
Lema 2.12 Sejam $\sigma_{1}, \sigma_{2}$ simpleros maximais de $\Lambda^{\prime}$. de dimensào maior ou igual a dois. tais que $\sigma_{1} \cap \sigma_{2}$ tem dimensào pelo menos um. Se $f:\left|K^{\prime}\right| \rightarrow|\Lambda|$ é uma aplicação com $a \in F i x(f)$ um ponto firo isolado $e$ se $b \in\left|\sigma_{1} \cap \sigma_{2}\right|$ é tal que Fix $(f) \cap[a, b]=\{a\}$ então triste $\Xi>0$ e uma aplicaçào $f^{\prime}:\left|K^{\prime}\right| \rightarrow\left|K^{\prime}\right|$ tais que:

(i) $f^{\prime} \simeq f \in f^{\prime}=f \in m\left|K^{\prime}\right| \backslash U([a, b] . \Xi)$;

(ii) Fix $\left(f^{\prime}\right) \cap \bar{U}([a, b] . \varepsilon)=\{c\}$ para algum $c \in\left|\sigma_{2}\right|$;

(iii) Se $z \in F i x(f), z \notin U([a, b], \varepsilon)$ e z está na mesma classe de ponto fixo da $f$ que a então z está na mesma classe de ponto fixo da $f^{\prime}$ que c.

Demonstração: [Brown, pg 136].

Introduziremos mais algumas convençòes para facilitar a linguagem.

Se $|K|$ é um poliedro conexo e $\sigma$ um simplexo de $K$ com $a \in|\sigma|, b \in \overline{|\sigma|}$ e $\bar{U}(a, \varepsilon) \subset|\sigma|$. definimos uma retraçào $R: \bar{U}([a, b], \Xi) \rightarrow[a, b]$ da seguinte forma:

Se $b \in|\sigma|$, tomamos $R(x)=x^{\prime} \in[a, b] \operatorname{com} d\left(x, x^{\prime}\right)=d(x,[a, b])$.

Se $b \in|\partial \sigma|$, dividimos em 3 situações:

(i) se $x \in[a, b]$ fazemos $R(x)=x$;

(ii) se $x \notin|\sigma|, R(x)=b$;

(iii) se $x \in|\sigma| \backslash[a, b]$ tomamos $y \in|\sigma|$ tal que, no plano determinado por $x$, $a \mathrm{e}$ $b$ a reta definida por $x$ e $y$ seja paralela a reta definida por $a$ e $b$. Escolhemos $\approx$ um ponto da reta $\stackrel{\leftrightarrow}{a b}$ de modo que $x, y, b, z$ seja um paralelograma. Definimos $R(x)=z$ se $z \in[a, b]$ e $R(x)=a$ se $z \notin[a, b]$.

Para $0<\eta<\varepsilon$ na situação colocada acima definimos

$$
W([a, b], \eta)=\{x \in \bar{U}([a, b], \Xi) \mid d(x, R(x))<\eta\} .
$$

Observamos que se $b \in|\sigma|$ temos que $W([a, b], \eta)=U([a, b], \eta)$.

Para $M \subset[a, b]$ definimos

$$
W([a, b], \eta, M)=\{x \in W([a, b] . \eta) \mid R(x) \in M\} .
$$

Denotaremos por $\bar{W}([a, b], \eta)$ o fecho de $W([a, b], \eta)$ e de forma semelhante o fecho de $W([a, b] . \eta . . W)$. 
Além dessas definições convencionamos que. se $x \in[a . b] .0 \leq t_{x} \leq 1$. é o único número. tal que $x=\left(1-t_{x}\right) a+t_{x} b$. Analogamente dado $t \in I . x_{t}$ é o único $x \in[a, b]$ tal que $x_{t}=(1-t) a+t b$.

Lema 2.13 Seja $\sigma$ um simplexo de $\mathrm{K}$ de dimensaio maior que um com a $\in|\sigma| \epsilon$ $b \in \overline{|\sigma|}$. Se existem aplicaçôs $f:\left|K^{-}\right| \rightarrow|\kappa| . H: I \times I \rightarrow|K| \epsilon M, N \subset[a, b]$ fechados tais que:

(1) $H(t, 0)=f\left(x_{t}\right) \quad \forall t \in I$;

(2) $H\left(t_{x}, u\right) \neq x \quad \forall x \in N, u \in I$;

(3) $H\left(t_{x}, u\right) \in \mathcal{V}(x) \forall x \in M, u \in I$.

Entioo existe uma aplicação $f^{\prime}:\left|K^{\prime}\right| \rightarrow\left|K^{\prime}\right|$ e um $\eta>0$ tais que:

(i) $f^{\prime} \simeq f$ e $f^{\prime}=f$ em $\left|K^{\prime}\right| \backslash W([a, b], \eta)$;

(ii) Fix $\left(f^{\prime}\right) \cap \bar{W}([a, b], \eta, N)=\emptyset$;

(iii) $f^{\prime}$ é próxima em $\bar{W}([a, b], \eta, M)$;

(iv) $f^{\prime}(x)=H\left(t_{x}, 1\right) \quad \forall x \in[a, b]$.

Demonstração: [Brown, pg 132].

Lema 2.14 Sejam $x \in\left|K^{\prime}\right|$ com $\sigma(x)$ maximal, uma aplicaçào $f:|K| \rightarrow\left|{ }^{\prime}\right| e$ $\eta>0$. Se $U(x, \eta) \subset|\sigma(x)|, F i x(f) \cap \partial U(x, \eta)=\emptyset$ ef é próxima $\epsilon m \bar{U}(x, \eta)$ então existe $f^{\prime}:\left|K^{\prime}\right| \rightarrow\left|K^{\prime}\right|$ tal que:

(i) $f^{\prime} \simeq f$ e $f^{\prime}=f$ em $\left|K^{\prime}\right| \backslash U(x, \eta)$;

(ii) Fix $\left(f^{\prime}\right) \cap \bar{U}(x, \eta)=\{x\}$;

(iii) f' é próxima em $\bar{U}(x, \eta)$.

Demonstração: [Brown. pg 126].

O lema a seguir é de fundamental importância pois realiza a mudança de posiçào de um ponto fixo isolado para qualquer outro ponto dado. Sua demonstração é. em grande parte. baseada na demonstraçào do lema 2.12 feita em [Brown]. 
Lema 2.15 Seja $|\hat{i}|$ um poliedro do tipo W. Se $f:\left|K^{\prime}\right| \rightarrow\left|K^{\prime}\right|$ é uma aplicaçào com $F i x(f)=\left\{a_{1}, a_{2}, \ldots, a_{r}\right\}$ e se $\sigma\left(a_{1}\right)$ é um simplexo maximal entào. dado $c \in\left|K_{i}\right|$. $c \notin$ Fir $(f)$. existe $f^{\prime} \simeq f$ com:

(i) Fir $\left(f^{\prime}\right)=\left\{c, a_{2}, \ldots, a_{r}\right\}$;

(ii) $f^{\prime}=f \in m$ uma vizinhança de' $\left\{a_{2}, \ldots, a_{r}\right\}$;

(iii) $\operatorname{ind}\left(f^{\prime}, c\right)=\operatorname{ind}\left(f, a_{1}\right)$.

Demonstração: Como $\left|K^{\prime}\right|$ é do tipo $\mathrm{W}$, tomamos $a_{1} \in|\sigma|$ e $c \in \overline{\left|\sigma^{\prime}\right|}$ simplexos maximais. Assim existe uma sequência $\sigma=\sigma_{1}, \sigma_{2}, \ldots, \sigma_{r}=\sigma^{\prime}$ de simplexos maximais com dimensão de $\left|\sigma_{i} \cap \sigma_{i+1}\right|$ maior que zero, $i=1, \ldots, r-1$.

Usando o lema 2.12 repetidas vezes, obtemos $g_{1} \simeq f \operatorname{com} F i x\left(g_{1}\right)=\left\{b, a_{2}, \ldots, a_{r}\right\}$ para algum $b \in\left|\sigma^{\prime}\right|$. Podemos obter $g_{1}$ de modo que $[b, c] \cap F i x(f)=\emptyset$ e $g_{1}=f$ em uma vizinhança de $\left\{a_{2}, \ldots, a_{r}\right\}$.

Tomamos $\delta>0$ tal que:

$$
\begin{gathered}
\bar{U}(b, 2 \delta) \subseteq\left|\sigma^{\prime}\right|, \\
\bar{U}(c, \delta) \subseteq|s t(\sigma(c))|, \\
F i x\left(g_{1}\right) \cap \bar{U}([b, c], \delta)=\{b\}, \\
f(\bar{U}(c, \delta)) \cap \bar{U}(c, \delta)=\emptyset,
\end{gathered}
$$

e

$$
f(\bar{U}(b, 2 \delta)) \subseteq\left|\overline{\sigma^{\prime}}\right|
$$

Escolhemos também $p, p^{\prime}, q \in[b, c]$ tais que:

$$
d(p, b)=\frac{\delta}{2}, \quad d\left(p^{\prime}, b\right)=2 \delta, \quad d(q, c)=\delta
$$

Sejam:

(i) $a(x)$ o ponto de intersecção da semi-reta $x \overrightarrow{f(x)}$ com $\partial \sigma^{\prime}$;

(ii) $\beta(x)$ o ponto de intersecção da semi-reta $q \overrightarrow{f(x)} \operatorname{com} \partial \sigma^{\prime}$;

(iii) sendo $\mathcal{A}$ um arco simples. contido em $\partial \sigma^{\prime}$, de um vértice $v$ de $\sigma^{\prime}$ até $\alpha(p)$; $h:[b, p] \rightarrow \mathcal{A}$ um homeomorfismo com $h(b)=\imath$ e $h(p)=\alpha(p)$. 
Assim podemos construir $g_{2}:[b, c] \rightarrow\left|K^{\prime}\right|$ definida por:

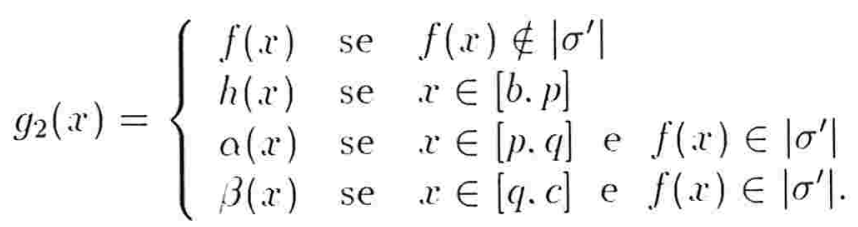

Como $g_{2}$ é contínua, temos que $g_{2}^{-1}(U(c, \delta))$ é aberto em $[b, c]$. Portanto

$$
g_{2}^{-1}\left([(c, \delta))=\bigcup\left(b_{i}, c_{i}\right),\right.
$$

onde cada $\left(b_{i}, c_{i}\right) \subset[b, c]$ é um intervalo aberto.

Como $[b, c]$ é compacto $g_{2}^{-1}(c)$ está contido em uma uniào finita, $\bigcup_{i=1}^{m}\left(b_{i}, c_{i}\right)$, desses intervalos.

Denotamos por $G_{r}^{\prime}$ o conjunto $L^{\prime}(c, \delta) \backslash\left|\sigma^{\prime}\right|$ observamos que, como $g_{2}([b, c]) \cap\left|\sigma^{\prime}\right|=\emptyset$, $g_{2}\left(\left(b_{j} . c_{j}\right)\right) \subset G$ para todo $j=1, \ldots, m$ e, além disso, $g_{2}\left(b_{j}\right), g_{2}\left(c_{j}\right) \in \partial G$.

$\bar{G}$ é contrátil, então podemos tomar $C_{j}:\left[b_{j}, c_{j}\right] \rightarrow \partial G$ homotópica à $g_{2}$ com $C_{j}(x) \neq b$ para todo $x \in\left[b_{j}, c_{j}\right]$ e $C_{j}\left(b_{j}\right)=g_{2}\left(b_{j}\right), C_{j}\left(c_{j}\right)=g_{2}\left(c_{j}\right)$.

Definimos então $g_{3}:[b, c] \rightarrow|K|$ por

$$
g_{3}(x)=\left\{\begin{array}{lll}
g_{2}(x) & \text { se } & x \notin \bigcup_{i=1}^{m}\left[b_{i}, c_{i}\right] \\
C_{j}(x) & \text { se } & x \in \bigcup_{i=1}^{m}\left[b_{i}, c_{i}\right]
\end{array} .\right.
$$

Definindo as homotopias $H_{2}:[b, c] \times I \rightarrow|K|, H_{1}:[b, c] \times I \rightarrow|K|$ e $H_{3}:$ $[b, c] \times I \rightarrow\left|I^{\prime}\right|$ tais que:

$$
\begin{gathered}
H_{2}(x, 0)=g_{2}(x) \text { e } H_{2}(x, 1)=g_{3}(x), \\
H_{1}(x, t)=(1-t) g_{1}(x)+t_{2}(x), \\
H_{3}(x, t)=g_{3}\left(x(1-t) t_{x}\right) .
\end{gathered}
$$

onde $x=\left(1-t_{x}\right) b+t_{x} c$, podemos construir $H:[b, c] \times I \rightarrow|\Lambda|$ dada por

$$
H(x, t)=\left\{\begin{array}{ll}
H_{1}(x, t) & \text { se } 0 \leq t \leq \frac{1}{3} \\
H_{2}(x, t) & \text { se } \frac{1}{3} \leq t \leq \frac{2}{3} \\
H_{3}(x, t) & \text { se } \frac{2}{3} \leq t \leq 1
\end{array} .\right.
$$

Verificamos que: 
(1) $H(x, 0)=g_{1}(x) \quad \forall x \in[b, c]$ :

(2) $H(x, t) \neq x \quad \forall x \in[p, c], t \in I$ :

(3) $H(x, t) \in \mathcal{V}(x) \forall x \in\left[b, p^{\prime}\right], t \in I$.

Usando o lema 2.13 para $M=\left[b, p^{\prime}\right]$ e $N=[p, c]$. obtemos $g_{4}:\left|K^{\prime}\right| \rightarrow|K|$ e $\eta>0$ tais que:

(i) $g_{4} \simeq g_{1}$ e $g_{4}=g_{1}$ em $|K| \backslash W([b, c], \eta)$;

(ii) Fir $\left(g_{4}\right) \cap \bar{W}([b, c], \eta,[p, c])=\emptyset$;

(iii) $g_{4}$ é próxima em $\bar{W}\left([b, c], \eta,\left[b, p^{\prime}\right]\right)$;

(iv) $g_{4}(x)=H(x, 1)=|v| \quad \forall x \in[b, c]$.

Além disso, na demonstração do lema 2.13, podemos obter $\eta$ de modo que

$$
W([b, c], \eta) \backslash W([b, c], \eta,[p, c]) \subset U(b, \delta),
$$

$g_{4}$ seja próxima em $W([b, c], \eta)$ e $F i x\left(g_{4}\right) \cap \partial U(b, \eta)=\emptyset$.

Aplicando, agora, o lema 2.14 obtemos $g_{5}$ tal que:

(i) $g_{5} \simeq g_{4}$ e $g_{5}=g_{4}$ em $\left|K^{\prime}\right| \backslash U(b, \eta)$;

(ii) Fix $\left(g_{5}\right) \cap U(b, \eta)=\{b\}$;

(iii) $g_{5}$ é próxima em $U(b, \eta)$.

Finalmente, basta definir $f^{\prime}:\left|K^{\prime}\right| \rightarrow\left|K^{\prime}\right|$ por

$$
f^{\prime}(x)= \begin{cases}g_{5}(x) & \text { se } x \notin L([b, c], \eta) \\ h\left(x, g_{5}(x),\left(1-t_{0}\right) \lambda+t_{0}\right) & \text { se } x=(1-\lambda) x_{\frac{\eta}{2}}+\lambda x_{\eta} \text { e } 0 \leq \lambda \leq 1 \\ (1-\lambda) c+\lambda h\left(x_{\frac{\eta}{2}}, g_{5}\left(x_{\frac{\eta}{2}}\right), t_{0}\right) & \text { se } x=(1-\lambda) c+\lambda x_{\frac{\eta}{2}} \text { e } 0 \leq \lambda \leq 1 \\ c & \text { se } x=c\end{cases}
$$

como no lema 2.8. para obtermos a aplicação desejada.

O resultado a seguir pode ser entendido como a não-essencialidade dos pontos fixos com índice zero. ou seja. podemos "eliminá-los". 
Lema 2.16 Seja $|\Lambda|$ um poliedro conexo. $\sigma$ um simplexo maximal de $K . f:\left|{ }^{\circ}\right| \rightarrow$ $\left|K^{*}\right|$ uma aplicação $x \in|\sigma|$ um ponto fixo isolado de $f \in U \subset|\sigma|$ uma vizinhanģa de $x$ tal que Fix $(f) \cap \bar{L}=\{x\}$. Se ind $(f, x)=0$ entrio. dado $\Xi>0$, existe uma aplicaçào $f^{\prime}:\left|K^{\prime}\right| \rightarrow\left|K^{\prime}\right|$ que satisfaz:

(i) $f=f^{\prime} \in m\left|H^{\prime}\right| \backslash l^{\prime}$ :

(ii) $d\left(f, f^{\prime}\right)<\Xi$;

(iii) Fix $(f) \cap \bar{U}=\emptyset$.

Demonstração: [Brown, pg 123].

Lema 2.17 Seja $f:|\kappa| \rightarrow|K|$ uma aplicaçào com $a \in F i x(f), \sigma(a)$ maximal $\epsilon$ $\operatorname{Fix}(f) \cap|\sigma(a)|=\{a\}$. Se $i_{1}, i_{2}, \ldots, i_{m}$ é uma sequência de inteiro tais que $\sum_{k=1}^{m} i_{k}=$ $\operatorname{ind}(f, a)$ então existe uma aplicação $f^{\prime}:\left|K^{\prime}\right| \rightarrow\left|K^{\prime}\right|$ tal que:

(i) $f^{\prime} \simeq f \in f^{\prime}=f \in m|K| \backslash|\sigma(a)|$;

(ii) Fix $\left(f^{\prime}\right) \cap|\sigma(a)|=\left\{a_{1}, a_{2}, \ldots, a_{m}\right\}$;

(iii) $\operatorname{ind}\left(f^{\prime}, a_{k}\right)=i_{k}$.

Ao contrário do lema anterior nosso objetivo aqui é "criar" novos pontos fixos de modo que satisfaçam condiçòes sobre seus índices. Note-se que esse resultado, somado ao lema 2.15 , serào nossas maiores ferramentas nos teoremas de localizaçào.

Demonstração: Seja $p$ a dimensão de $\sigma(a)$.

Escolhemos $\varepsilon>0$ de modo que $\bar{U}(a, 5 \varepsilon) \subset|\sigma(a)|$. Tomamos $0<\delta<\varepsilon$ tal que $f(\bar{U}(a, \delta)) \subset \bar{L}(a, \bar{\varepsilon})$.

Denotamos por $B$ o disco $\bar{U}(a, \delta)$. Sejam $a_{1}, a_{2}, \ldots, a_{m}$ pontos de $B$ tais que $d\left(a, a_{k}\right)$ é constante e exista $\rho>0 \operatorname{com} \delta-d\left(a, a_{k}\right)<\rho<d\left(a, a_{k}\right)$ e $\bar{U}\left(a_{k}, \rho\right) \cap \bar{U}\left(a_{j}, \rho\right)=\emptyset$ se $k \neq j$.

Fazendo $A_{k}=B \cap \bar{L}\left(a_{k}, \rho\right)$, temos que $A_{k}$ é homeomorfo ao disco $D^{p}$ e que $A_{k} \cap \partial B \neq \emptyset$ (mais especificamente homeomorfo a $D^{p-1}$ ).

Tomamos $h_{k}:\left(\mathbb{R}^{n} . \sigma(a), a_{k}\right) \rightarrow\left(\mathbb{R}^{n}, \mathbb{R}^{p}, 0\right)$ uma isometria que leva $a_{k}$ na origem e o $p$-subespaço que contém $\sigma(a)$ em $\mathbb{R}^{p}$. 
Definimos $d_{k}^{\prime}: \partial A_{k} \cap \partial B \rightarrow \mathbb{R}^{p} \backslash\{0\}$ por

$$
d_{k}^{\prime}(y)=h_{k}(y)-h_{k}(f(y)) .
$$

Observando que $\left\|d_{k}^{\prime}(y)\right\| \leq\left\|h_{k}(y)\right\|+\left\|h_{k} \circ f(y)\right\| \leq \rho+\varepsilon+\delta \leq 3 \varepsilon$, podemos escrever

$$
d_{k}^{\prime}: \partial A_{k} \cap \partial B \rightarrow \bar{U}(0,3 \Sigma) \backslash\{0\} .
$$

Como $\partial A_{k} \cap \partial B$ é contrátil e o par $\left(\partial A_{k}, \partial A_{k} \cap \partial B\right)$ tem a propriedade cla extensào homotópica temos que, escolhidos geradores apropriados $\alpha$ e $\mu$ de $H_{p-1}\left(\partial A_{k}\right.$ ) e $H_{p-1}(\bar{U}(0,3 \varepsilon) \backslash\{0\})$, existe $d_{k}: \partial A_{k} \rightarrow \bar{U}(0,3 \varepsilon) \backslash\{0\}$, extensão de $d_{k}^{\prime}$ tal que

$$
d_{k *}(\alpha)=i_{k} \cdot \mu \text {. }
$$

Assim podemos definir:

$$
g_{k}^{\prime}(y)=h_{k}^{-1}\left(h_{k}(y)-d_{k}(y)\right) \quad \forall y \in \partial A_{k} .
$$

Como $\left\|h_{k} \circ g_{k}^{\prime}(y)\right\| \leq\left\|h_{k}(y)\right\|+\left\|d_{k}(y)\right\| \leq \delta+3 \varepsilon<4 \varepsilon$ e $d\left(a, g_{k}^{\prime}(y)\right) \leq d\left(a, a_{k}\right)+$ $d\left(a_{k}, g_{k}^{\prime}(y)\right) \leq \delta+4 \varepsilon<5 \varepsilon$ temos que

$$
g_{k}^{\prime}: \partial A_{k} \rightarrow|\sigma(a)|
$$

Como $A_{k}$ é homeomorfo a $D^{p}$, extendemos $g_{k}^{\prime}$ para $g_{k}: A_{k} \rightarrow|\sigma(a)|$ por linearidade (usando a projeção com centro em $a_{k}$ ) de modo que $F i x\left(g_{k}\right)=\left\{a_{k}\right\}$. $i_{k}$.

É importante salientar que $g_{k}$ coincide com $f$ em $\partial B \cap A_{k}$, além disso ind $\left(g_{k}, a_{k}\right)=$

Definimos então $g^{\prime}: \bigcup_{k=1}^{m} A_{k} \cup \partial B \rightarrow|\sigma(a)|$ dada por

$$
g^{\prime}(x)=\left\{\begin{array}{lll}
g_{k}(x) & \text { se } & x \in A_{k} \\
f(x) & \text { se } & x \in \partial B
\end{array}\right.
$$

Novamente vamos extender $g^{\prime}$ em $B$ por linearidade pois $B \backslash \bigcup_{k=1}^{m} A_{k}$ é homeomorfo a $D^{p}$ e $g^{\prime}$ está definida em $\partial\left(B \backslash \bigcup_{k=1}^{m} A_{k}\right)$.

Obtemos assim $g: B \rightarrow|\sigma(a)| \operatorname{com} g=g^{\prime} \mathrm{em}\left(\bigcup_{k=1}^{m} A_{k}\right) \bigcup \partial B$ e Fir $(g)=$ $\left\{a . a_{1}, \ldots . a_{m}\right\}$. 
Tomando $f_{1}:\left|K^{\prime}\right| \rightarrow\left|K^{\prime}\right|$ satisfazendo

$$
f_{1}(x)=\left\{\begin{array}{lll}
f(x) & \text { se } & x \in|K| \backslash B \\
g(x) & \text { se } & x \in B
\end{array}\right.
$$

temos que $f_{1}$ é contínua. $f_{1} \simeq f, f_{1}=f$ em $|K| \backslash B . F i x\left(f_{1}\right) \cap|\sigma(a)|=\left\{a, a_{1}, a_{2}, \ldots, a_{m}\right\}$ $\mathrm{e} \operatorname{ind}\left(f_{1}, a_{k}\right)=i_{k}$.

Sabendo que $\operatorname{ind}\left(f,\left|K^{\prime}\right|\right)=\operatorname{ind}\left(f_{1},\left|\Lambda^{-}\right|\right)$(pois $f_{1} \simeq f$ ) e, usando a propriedade aditiva do índice, temos que

$$
\operatorname{ind}\left(f_{1}, a\right)+\sum_{k=1}^{m} \operatorname{ind}\left(f_{1} \cdot a_{k}\right)=\operatorname{ind}(f, a) .
$$

Portanto

$$
\operatorname{ind}\left(f_{1}, a\right)=0 \text {. }
$$

Tomamos $0<\delta^{\prime}<\varepsilon^{\prime}<d\left(a, a_{k}\right)$ tais que $\bar{U}\left(a, 2 \Xi^{\prime}\right) \subset|\sigma(a)|$ e $f_{1}\left(\bar{U}\left(a, 2 \delta^{\prime}\right)\right) \subset$ $\bar{U}\left(a, 2 \Sigma^{\prime}\right)$ e, usando o lema 2.16 , obtemos uma aplicação $f^{\prime}:\left|K^{\prime}\right| \rightarrow\left|K^{\prime}\right|$ tal que

(i) $f^{\prime}=f_{1}$ em $\left|K^{\prime}\right| \backslash \bar{U}\left(a, 2 \delta^{\prime}\right)$;

(ii) $d\left(f_{1}, f^{\prime}\right)<\varepsilon^{\prime}$ (portanto $\left.f^{\prime} \simeq f_{1}\right)$;

(iii) Fix $\left(f^{\prime}\right) \cap \bar{U}\left(a, \delta^{\prime}\right)=\emptyset$.

$f^{\prime}$ é a aplicação desejada.

A partir de agora apresentaremos alguns resultados. O primeiro deles é uma aplicaçào quase imediata dos lemas que construímos até aqui.

Teorema 2.18 Sejam $c_{k}$ pontos em um poliedro $\left|K^{\prime}\right|$ do tipo $W$ e inteiros $i_{k}$ com $k=1, \ldots, m$. Existe uma deformaçào $f:\left|\Lambda^{\dagger}\right| \rightarrow\left|K^{\prime}\right| \operatorname{com} F i x(f)=\left\{c_{1}, \ldots, c_{k}\right\} \epsilon$ $\operatorname{ind}\left(f, c_{k}\right)=i_{k}$ se, $\epsilon$ somente se, $\sum_{k=1}^{m} i_{k}=\backslash(|K|)$.

Demonstração: $(\Leftarrow)$ A necessidade de $\sum_{k=1}^{m} i_{k}=\backslash\left(\left|K^{\prime}\right|\right)$ decorre do fato de que se $f \simeq$ id entào $L(f)=\backslash\left(\left|\kappa^{\prime}\right|\right)$.

$\left(\Rightarrow\right.$ ) Usando o teorema 2.9 para $A$ um ponto $a \neq a_{k} \cdot 1 \leq k \leq m, \operatorname{com} \sigma(a)$ maximal. obtemos $f_{1}:\left|K^{\prime}\right| \rightarrow\left|K^{\prime}\right|$ uma deformação tal que Fix $\left(f_{1}\right)=\{a\}$ e portanto $\operatorname{ind}\left(f_{1}, a\right)=\backslash(|\Lambda|)$. 
Se preciso. subdividimos $\left|K^{i}\right|$ para que $\overline{\sigma(a)} \cap\left\{c_{1}, \ldots, c_{m}\right\}=\emptyset$. Usando o lema $2.1 \bar{\imath}$ obtemos $f_{2}:\left|K^{i}\right| \rightarrow\left|K^{i}\right| \operatorname{com} F i x\left(f_{2}\right)=\left\{a_{1} \ldots a_{m}\right\} \subset|\sigma(a)|$ e $\operatorname{ind}\left(f_{2}, a_{k}\right)=i_{k}$.

Usando $m$ vezes o lema 2.15 conseguimos a aplicação $f:|\Lambda| \rightarrow|\Lambda| \operatorname{com} f \simeq i d$. Fix $(f)=\left\{c_{1}, \ldots, c_{m}\right\}$ e ind $\left(f, c_{k}\right)=i_{k}$.

Para estender esse resultado no caso de $f$ nào ser uma deformaçào, vamos precisar de um teorema que, sob determinadas condiçòes. garanta a realizaçào de $N(f)$ como o número exato de pontos fixos de alguma $f^{\prime} \simeq f$.

Definição $2.19 \operatorname{lm}$ poliedro $|K|$ é do tipo $\mathrm{S}$ se a dimensão de $K$ é maior ou igual a três e se para todo vértice $v$ de $|K|$, dst $(v)$ é conexo.

Teorema 2.20 Seja $\left|K^{\prime}\right|$ um poliedro do tipo $S$, se $f:\left|K^{\prime}\right| \rightarrow\left|K^{\prime}\right|$ é contínua então existe $f^{\prime} \simeq f$ tal que \#Fix $\left(f^{\prime}\right)=N(f)$ e $\sigma(x)$ é maximal para todo $x \in F i x\left(f^{\prime}\right)$.

Demonstração: [Brown, pg 140].

Agora podemos enunciar um teorema de localização mais geral.

Teorema 2.21 Sejam $|K|$ um poliedro do tipo $S$, uma aplicação $f:|K| \rightarrow|K|$ com classes de ponto fixo essenciais $\mathbb{F}_{l}, c_{1}, \ldots, c_{m}$ pontos de $|K|$ e $i_{1}, \ldots, i_{m}$ inteiros. Existe $f^{\prime} \simeq f$ com Fix $\left(f^{\prime}\right)=\left\{c_{1}, \ldots, c_{m}\right\}$ e ind $\left(f^{\prime}, c_{k}\right)=i_{k}$ se, e somente se, $c_{k}$ pode ser reindexado $c_{j}$ com $1 \leq j=j(k) \leq m$ de modo que: ou $N(f)=0$ e $\sum_{j=1}^{m} i_{j}=0$ ou existe uma sequência de inteiros $m_{0}, \ldots, m_{N(f)} \operatorname{com} 0=m_{0}<m_{1}<. .<m_{N(f)}=m$ que satisfa $\sum_{j=m_{l-1}+1}^{m_{l}} i_{j}=\operatorname{ind}\left(f, \mathbb{F}_{l}\right) \operatorname{para} l=1, \ldots, N(f)$.

\section{Demonstração: $(\Rightarrow)$ Trivial.}

$(\Leftarrow)$ Se $N(f)>0$ usamos o teorema 2.20 e obtemos $f_{1}:\left|K^{\prime}\right| \rightarrow\left|{ }^{\prime}\right|, f_{1} \simeq f \mathrm{com}$ exatamente $N(f)$ pontos fixos.

Subdividindo $|K|$, se necessário, podemos usar o lema 2.15 para construir $f_{2} \simeq f_{1}$ $\operatorname{com} \operatorname{Fix}\left(f_{2}\right)=\left\{a_{1}, \ldots, a_{N(f)}\right\}, \sigma\left(a_{l}\right) \cap\left\{a_{1}, \ldots a_{N(f)}, c_{1}, . . c_{m}\right\}=\left\{a_{l}\right\}$ e $\operatorname{ind}\left(f_{2}, a_{l}\right)=$ ind $\left(f, F_{l}\right)$.

Com o lema 2.17 "transformamos" cada ponto fixo $a_{l}$ de $f_{2} \mathrm{em} m_{l}-m_{l-1}$ pontos fixos de uma aplicação $f_{3}:\left|K^{\prime}\right| \rightarrow\left|K^{\prime}\right|$ de modo que $F i x\left(f_{3}\right)=\left\{b_{1}, \ldots, b_{m}\right\}$ e $\operatorname{ind}\left(f_{3}, b_{j}\right)=i_{j}$. 
Usando o lema 2.15 novamente "levamos" cada $b_{j}$ no ponto fixo $c_{j}$ de uma $f^{\prime}$ que é a aplicaçào desejada.

Se $N(f)=0$ pelo teorema 2.20 existe $f_{1}:|K| \rightarrow|\Lambda|$ com Fir $\left(f_{1}\right)=\emptyset$. Se conseguirmos $f_{2}:\left|K^{\prime}\right| \rightarrow\left|K^{\prime}\right| f_{2} \simeq f_{1} \operatorname{com} F i x\left(f_{2}\right)=\{a\}, \sigma(a)$ maximal encerramos a demonstraçào repetindo o argumento usado na demonstração do teorema 2.18.

Para tanto. subdividindo $\left|K^{\dagger}\right|$ se preciso. escolhemos $a \in\left|K^{i}\right|$ com $\sigma(a)$ maximal de modo que $\sigma(a) \cap\left\{c_{1}, \ldots . c_{m}\right\}=\emptyset$ e exista $\eta>0$ tal que

$$
\begin{gathered}
\bar{U}(a, \eta) \subset \sigma(a), \\
f_{1}(\bar{U}(a, \eta)) \subset \operatorname{st}\left(\sigma\left(f_{1}(a)\right)\right)
\end{gathered}
$$

$\mathrm{e}$

$$
\bar{U}(a, \eta) \cap \operatorname{st}\left(\sigma\left(f_{1}(a)\right)\right)=\emptyset .
$$

Tomamos $\gamma: I \rightarrow|K|$ uma curva de $a$ até $f_{1}(a) \operatorname{com} d(a, \gamma(t)) \neq \frac{t \eta}{2}$ se $t \neq 0$.

Sendo $\zeta: \bar{U}(a, \eta) \backslash\{a\} \rightarrow \partial \bar{U}(a, \eta)$ e $\zeta_{\frac{1}{2}}: \bar{U}\left(a, \frac{\eta}{2}\right) \backslash\{a\} \rightarrow \partial \bar{U}\left(a, \frac{\eta}{2}\right)$ as projeções com centro em $a$, definimos $f_{2}:\left|K^{\prime}\right| \rightarrow\left|K^{\prime}\right|$ por:

$$
f_{2}(x)= \begin{cases}f_{1}(x) & \text { se } x \in|\zeta| \backslash \bar{U}(a, \eta) \\ t \cdot f_{1}(\zeta(x))+(1-t) \cdot f_{1}(a) & \text { se } x=t \zeta(x)+(1-t) \zeta_{\frac{1}{2}}(x) \quad 0 \leq t \leq 1 \\ \gamma(t) & \text { se } x=t \zeta \frac{1}{2}(x)+(1-t) a \quad 0 \leq t \leq 1 .\end{cases}
$$

Temos que $f_{2} \simeq f_{1}$ e Fix $\left(f_{2}\right)=\{a\}$ com $\sigma(a)$ maximal.

O último resultados que apresentaremos neste capítulo é o análogo do teorema 2.21 para a esfera $S^{2}$, que nào é um poliedro do tipo $S$ mas, por ser simplesmente conexa, só admite uma classe de ponto fixo.

Teorema 2.22 Se $c_{1}, \ldots, c_{m}$ são pontos $e m S^{2} \in i_{1}, \ldots, i_{m}$ são inteiros então triste uma aplicaçào $f: S^{2} \rightarrow S^{2} \operatorname{com} F i x(f)=\left\{c_{1}, \ldots, c_{m}\right\} \in \operatorname{ind}\left(f, c_{k}\right)=i_{k}$.

Demonstração: Vamos identificar $S^{\prime 2} \mathrm{com}$ a suspensào do círculo $\|z\|=1$ no plano complexo. dessa forma se $x \in S^{2}$ temos $x=(z, s)$ com $z \in S^{1} \subset \mathbb{C}$ e $s \in[-1,1]$.

Tomamos $d=-1+\sum_{k=1}^{m} i_{k}$ e $\lambda:[-1,1] \rightarrow \mathbb{R}$ dada por

$$
\lambda(s)=\left\{\begin{array}{lll}
\frac{1+s}{2} & \text { se }-1 \leq s \leq 0 \\
\frac{1-s}{2} & \text { se }-\leq s \leq 1
\end{array}\right.
$$


e definimos $f_{0}: S^{2} \rightarrow S^{2} \mathrm{com}$

$$
f_{0}((z, s))=\left(z^{d} \cdot s+\lambda(s)\right) .
$$

Assim $f_{0}$ tem grau d e Fix $\left(f_{0}\right)=\{(z,-1),(z, 1)\}$.

Escolhemos $a=\left(z_{0}, 0\right)$ de modo que $a \notin\left\{c_{1}, \ldots . c_{m}\right\}$ e $z_{0}^{d} \neq-z_{0}$.

Denotamos por $\gamma(t) \quad 0 \leq t \leq 1$. o único arco de $\zeta^{2}$ que começa em $(z, 1)$, termina em $(z,-1)$ e passa por $a$.

Tomamos $\delta>0$ e $\varepsilon>0$ satisfazendo

(1) $\left\|z-z_{0}\right\| \leq \delta \Rightarrow z^{d} \neq-z_{0}$;

(2) $\left(z,-\frac{1}{3}\right) \in \bar{U}(\gamma, \varepsilon) \Rightarrow\left\|z-z_{0}\right\|<\delta$;

(3) $\left(z_{0}, 0\right) \notin \bar{L}(\gamma, \varepsilon)$.

Assim

$$
f_{0}(x) \neq-a \quad \forall x \in \bar{U}(\gamma, \varepsilon) .
$$

Seja $y(x): \bar{U}(\gamma, \varepsilon) \rightarrow \partial \bar{U}(\gamma, \varepsilon)$ a projeção com centro em $a$.

Temos que se $x \in \bar{U}(\gamma, \varepsilon) \backslash\{a\}$ então $x=(1-t) a+t y(x)$ para algum $t$ com $0<t \leq 1$.

Definimos $f_{1}: S^{2} \rightarrow S^{2}$ por

$$
f_{1}(x)= \begin{cases}f_{0}(x) & \text { se } x \in S^{2} \backslash \bar{U}(\gamma, \Xi) \\ (1-t) a+t f_{0}(y(x)) & \text { se } x \in \bar{U}(\gamma, \Xi), \quad x=(1-t) a+t y(x) \\ a & \text { se } x=a\end{cases}
$$

e verificamos que:

(i) $f_{1} \simeq f_{0}$

(ii) $\operatorname{Fix}\left(f_{1}\right)=\{a\}$

(iii) ind $\left(f_{1}, a\right)=\sum_{k=1}^{m} i_{k}$ pois ind $\left(f_{1}, a\right)=\operatorname{ind}\left(f_{1}, S^{2}\right)=L\left(f_{1}\right)=1+d$.

Em resumo obtemos uma aplicação de $S^{2} \mathrm{em} S^{2}$ com exatamente um ponto fixo com índice igual a soma dos inteiros da sequência dada. C'omo $S^{2}$ é do tipo $\mathrm{W}$ podemos usar os lemas 2.17 e 2.15. 


\section{Capítulo 3}

\section{Localização de Coincidências}

Vesse capítulo vamos apresentar alguns resultados de localização de coincidências semelhantes aos obtidos no capítulo 2 para pontos fixos.

Usando a notação:

$$
I=\left\{\left(x_{1}, x_{2}, . ., x_{n}\right) \in \mathbb{R}^{n} \mid 0 \leq x_{1} \leq 1, x_{j}=0 j=2, \ldots, n\right\}
$$

$\mathrm{e}$

$$
V^{n}=\left\{x \in \mathbb{R}^{n} \mid d(x, I) \leq 1\right\},
$$

podemos enunciar:

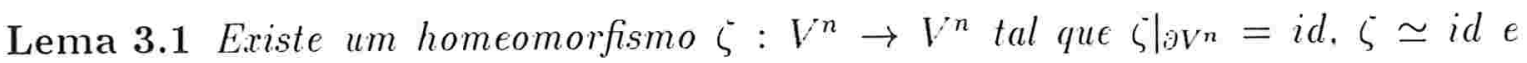
$\zeta(0, \ldots .0)=(1,0, \ldots, 0)$.

Demonstração: Para cada $x \in V^{n}, x \neq 0$ existe um único $x_{\partial} \in \partial V^{n}$ tal que $x=t_{x} x_{\partial}, \quad 0<t_{x} \leq 1$ ( $x_{\partial}$ o ponto de intersecção de $\partial V^{n}$ com a semi-reta de início na origem e que contém $x$ ).

Definimos $\zeta: V^{n} \rightarrow V^{n}$ por

$$
\zeta(x)= \begin{cases}(1,0, \ldots, 0) & \text { se } x=0 \\ t_{x} x_{\partial}+\left(1-t_{x}\right)(1,0 \ldots 0) & c c .\end{cases}
$$

Claramente $\left.\zeta\right|_{\partial V^{n}}=i d$ e $\zeta \simeq i d$ (basta tomar $\left.H(x, t)=(1-t) x+t \zeta(x)\right)$. 


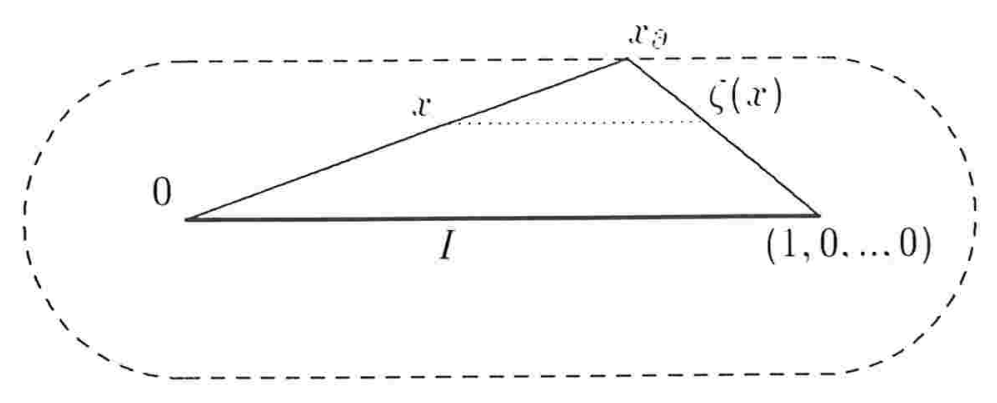

Os próximos dois lemas reproduzem, para coicidências, os principais lemas do capítulo anterior.

Lema 3.2 Dados $X$, variedade $n$-dimensional $(n \geq 2)$, conera, orientável $\epsilon$ sem bordo. Y um poliedro e aplicações $f_{1}, f_{2}: X \rightarrow Y \operatorname{com} \operatorname{Coin}\left(f_{1}, f_{2}\right)=\left\{a_{1}, a_{2}, \ldots, a_{r}\right\}$ e $p \in X$ tal que $p \notin C$ Coin $\left(f_{1}, f_{2}\right)$. Então existe um par $\left(g_{1}, g_{2}\right)$ homotópico a $\left(f_{1}, f_{2}\right)$ tal que $\operatorname{Coin}\left(g_{1}, g_{2}\right)=\left\{p, a_{2}, \ldots, a_{r}\right\}$; além disso $g_{i}=f_{i}, i=1,2$, em uma vizinhança de $\left\{a_{2}, \ldots, a_{r}\right\}$.

Demonstração: Tomamos $C \subset \mathrm{X}$ uma curva simples de $p$ até $a_{1}$. É fácil ver que podemos tomar $C$ de modo que exista uma vizinhança $U$ de $C$ tal que $\bar{U} \cap$ $\operatorname{Coin}\left(f_{1}, f_{2}\right)=\left\{a_{1}\right\}$ e $\bar{U}$ é homeomorfo, por $\varphi$, a $V^{n} ; \operatorname{com} \varphi(p)=0, \varphi\left(a_{1}\right)=(1,0, \ldots, 0)$ e $\varphi(C)=I$.

Definimos:

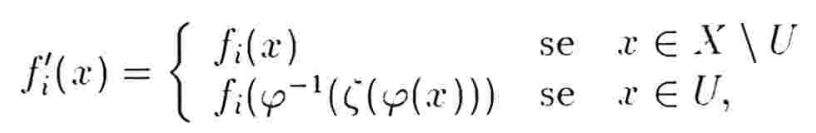

$i=1,2$ com $\zeta$ dada pelo lema 3.1 .

Temos então que $f_{i}^{\prime} \simeq f_{i}$ pois $\zeta \simeq i d, C \operatorname{oin}\left(f_{1}^{\prime}, f_{2}^{\prime}\right)=\left\{p, a_{1}, a_{2}, \ldots, a_{r}\right\}$ e $\left.f_{i}^{\prime}\right|_{X \backslash \overline{U^{\prime}}}=$ $\left.f_{i}\right|_{X \backslash \bar{C}}$.

Lema 3.3 Seja $\left(f_{1}, f_{2}\right): X \rightarrow Y$ um par de aplicaçòes entre variedades n-dimensionais $n \geq 2$. coneras. Se a é uma coincidência isolada de $f_{1}$ e $f_{2} \operatorname{com} \operatorname{ind}\left(f_{1}, f_{2}, a\right)=k \epsilon$ $i_{1}, \ldots . i_{r}$ é uma sequência de inteiros com $\sum_{j=1}^{r} i_{j}=k$ então existem $U \subset X$ e um par $\left(f_{1}^{\prime}, f_{2}^{\prime}\right)$ homotópico à $\left(f_{1}, f_{2}\right)$ tais que:

(i) $\operatorname{Coin}\left(f_{1}, f_{2}\right) \cap \bar{l}=\{a\}$ : 
(ii) $\left.f_{j}^{\prime}\right|_{X \backslash C^{\circ}}=\left.f_{j}\right|_{X \backslash U} j=1,2$ :

(iii) $\operatorname{Coin}\left(f_{1}^{\prime}, f_{2}^{\prime}\right) \cap U=\left\{a_{1}, \ldots, a_{r}\right\} \quad \in \operatorname{ind}\left(f_{1}^{\prime} \cdot f_{2}^{\prime} \cdot a_{j}\right)=i_{j}$.

Demonstração: Tomamos $U \subset X$ aberto. $\operatorname{com} \operatorname{Coin}\left(f_{1}, f_{2}\right) \cap U=\{a\}$ e $\bar{l}$ homeomorfo, por $\hat{\varphi}$. a $B^{n}=\left\{x \in \mathbb{R}^{n} \mid\|x\| \leq 1\right\}$, $\hat{\gamma}(a)=0$, de modo que $f_{1}\left(\overline{l^{C}}\right) \cup$ $f_{2}(\bar{U}) \subset V$ com $V$ aberto de $Y, \bar{V}$ homeomorfo, por $\psi$ à $B^{n}, \psi\left(f_{1}(a)\right)=0$. Além disso tomamos $U$ e $V$ com $\psi\left(f_{1}(\bar{U}) \cup f_{2}(\bar{U})\right) \subset B_{\frac{1}{2}}^{n} . \quad B_{\frac{1}{2}}^{n}=\left\{x \in \mathbb{R}^{n} \mid\|x\| \leq \frac{1}{2}\right\}$ e $\left\|\psi\left(f_{1}(x)\right)-\psi\left(f_{2}(x)\right)\right\|<\frac{1}{2}$.

Identificando os pontos de $\bar{U}$ e de $\bar{V}$ com os de $B^{n}$, trataremos $f_{1}$ e $f_{2}$ como aplicaçòes de $B^{n}$ em $B^{n}$. Dessa maneira definimos $h: B^{n} \rightarrow B_{\frac{1}{2}}^{n}$ por

$$
h(x)=f_{2}(x)-f_{1}(x) .
$$

Como $h(x) \neq 0 . \forall x \in \partial B^{n}$ temos $\left.h\right|_{\partial B^{n}}: S^{n} \rightarrow B_{\frac{1}{2}}^{n} \backslash 0$.

Pela proposição 1.25 sabemos que $\operatorname{ind}\left(f_{1}, f_{2}, a\right)=\operatorname{deg}\left(\left.h\right|_{\partial B^{n}}\right)$.

Tomamos $a_{1}, . . a_{r} \in B^{n}$ tais que exista $\rho>0$ com $\left\|a_{j}\right\|>1-\rho$ e $\left\|a_{j}\right\|>\rho, \forall a_{j} \mathrm{e}$ $B_{\rho}\left(a_{j}\right) \cap B_{\rho}\left(a_{k}\right)=\emptyset$ se $i \neq k$, com $B_{\rho}\left(a_{j}\right)=\left\{x \in \mathbb{R}^{n} \mid\left\|x-a_{j}\right\| \leq \rho\right\}$.

Fazendo $A_{j}=B^{n} \cap B_{\rho}\left(a_{j}\right)$, temos $\left.h\right|_{\partial B^{n}}$ definida em $\partial A_{j} \cap \partial B^{n}$ com imagem em $B_{\frac{1}{2}}^{n} \backslash\{0\}$.

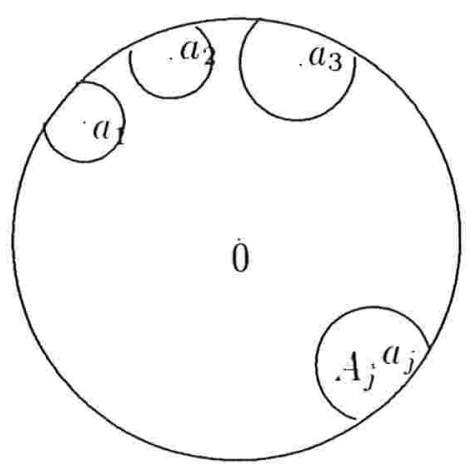

Observando que $A_{j}$ é homeomorfo à $B^{n}$ temos que $\left(\partial A_{j}, \partial A_{j} \cap \partial B^{n}\right)$ tem a propriedade da extensão homotópica em relação à $B_{\frac{1}{2}}^{n} \backslash\{0\}$. Como $\partial A_{j} \cap \partial B^{n}$ é contrátil existe $h_{j}: \partial A_{j} \rightarrow B_{\frac{1}{2}}^{n} \backslash\{0\}$ extensão de $\left.h\right|_{\partial A_{j} \cap B^{n}} \operatorname{com} \operatorname{deg}\left(h_{j}\right)=i_{j}$.

Definimos $h_{j}$ no interior de $A_{j}$ por linearidade. usando o homeomorfismo entre $t_{j}$ e $B^{n}$. de modo que $h_{j}(x)=0 \Leftrightarrow x=a_{j}$, pois $h_{j}(x) \neq 0 . \forall x \in \partial A_{j}$. 
Desse modo podemos definir $h^{\prime}: \partial B \cup A_{j} \rightarrow B_{\frac{1}{2}}^{n}$ por:

$$
h^{\prime}(x)=\left\{\begin{array}{lll}
h(x) & \text { se } & x \in \partial B \\
h_{j}(x) & \text { se } & x \in A_{j}
\end{array}\right.
$$

Definimos $h^{\prime}$ no interior de $B^{n} \backslash \bigcup_{j=1}^{r} A_{j}$ de modo análogo ao que fizemos para $h_{j}$. Temos que: $h^{\prime}(x)=0 \Leftrightarrow x \in\left\{0, a_{1}, a_{2}, \ldots, a_{r}\right\}$.

Definindo $f_{1}^{\prime \prime}: B^{n} \rightarrow B^{n}$ por:

$$
f_{1}^{\prime \prime}(x)=f_{2}(x)-h^{\prime}(x) .
$$

temos que $f_{1}^{\prime \prime} \simeq f_{2}$ rel $\partial B^{n}$ (basta tomar $\left.H(x, t)=t f_{1}(x)+(1-t) f_{1}^{\prime \prime}\right)$.

Assim.

$$
\sum_{j=1}^{r} \operatorname{ind}\left(f_{1}^{\prime \prime}, f_{2}, a_{j}\right)+\operatorname{ind}\left(f_{1}^{\prime \prime}, f_{2}, a\right)=\operatorname{ind}\left(f_{1}, f_{2}, a\right)=k
$$

Mas

$$
\sum_{j=1}^{r} \operatorname{ind}\left(f_{1}^{\prime \prime}, f_{2}, a_{j}\right)=\sum_{j=1}^{r} i_{j}=k
$$

Portanto ind $\left(f_{1}^{\prime \prime}, f_{2}, a\right)=0$.

Assim sendo, observando que $\partial\left(B^{m} \backslash \bigcup_{j=1}^{r} A_{j}\right)$ é homeomorfo à $B^{n}$, temos

$$
\operatorname{deg}\left(\left.h^{\prime}\right|_{\partial\left(B \backslash \bigcup_{j=1}^{r} A_{j}\right)}\right)=0
$$

Identificando $B \backslash \bigcup_{j=1}^{r} A_{j} \operatorname{com} B^{n}$, e restringindo $h^{\prime}$ de modo conveniente obtemos $h^{\prime}: S^{n-1} \rightarrow B_{\frac{1}{2}}^{n} \backslash\{0\}$ homotópica a constante.

Para cada $x \in \overline{\left(B^{n} \backslash B_{\frac{1}{2}}^{n}\right)}$ temos $x=\left(1-t_{x}\right) x_{1}+t_{x} x_{\frac{1}{2}}$ onde $0 \leq t_{x} \leq 1, x_{1} \in \partial B^{n}$ e $x_{\frac{1}{2}} \in \partial B_{\frac{1}{2}}^{n}\left(x_{1}, x_{\frac{1}{2}}\right.$ imagens de $x$ pela projeção central).

Definimos $h^{\prime \prime}: B^{n} \rightarrow B^{n}$ por:

$$
h^{\prime \prime}(x)=\left\{\begin{array}{lll}
H\left(x_{1}, t_{x}\right) & \text { se } & x \in \overline{\left(B^{n} \backslash B_{\frac{1}{2}}^{n}\right)} \\
H\left(x_{1}, 1\right) & \text { se } & x \in B_{\frac{1}{2}}^{n}
\end{array},\right.
$$


onde $H(x, t): S^{n-1} \times I \rightarrow B_{\frac{1}{2}}^{n} \backslash\{0\}$ é a homotopia entre $h^{\prime}$ e a aplicaçào constante. Assim sendo, usando novamente a identificaçào de $B \backslash \bigcup_{j=1}^{r} A_{j} \operatorname{com} B^{n}$ defino $h^{\prime \prime}$ no seu interior $\operatorname{com} h^{\prime \prime}(x) \neq 0, \forall x$.

Fazendo

$$
f_{1}^{\prime \prime \prime}(x)=f_{2}(x)-h^{\prime \prime}(x) .
$$

temos que $f_{1}^{\prime \prime \prime} \simeq f_{1}$ rel $\partial B^{n}$ e $C \operatorname{oin}\left(f_{1}^{\prime \prime \prime}, f_{2}\right)=\left\{a_{1}, \ldots a_{r}\right\}$. Além disso ind $\left(f_{1}^{\prime \prime \prime}, f_{2}, a_{j}\right)=$ $i_{j}$.

Usando mais uma vez a identificação de $\bar{U} \operatorname{com} B^{n}$ construimos $f_{1}^{\prime}: X \rightarrow Y$ satisfazendo

$$
f_{1}^{\prime}(x)=\left\{\begin{array}{lll}
f(x) & \text { se } & x \in X \backslash U \\
f_{1}^{\prime \prime \prime}(x) & \text { se } & x \in U
\end{array}\right.
$$

O par $\left(f_{1}^{\prime}, f_{2}\right)$ satisfaz as condições exigidas.

Observamos que na demostraçào acima obtemos um resultado básico da teoria que poderia ser demonstrado, sem maiores dificuldades, de maneira direta.

Corolário 3.4 Se a $\in \operatorname{Coin}\left(f_{1}, f_{2}\right)$ é uma coincidência isolada e ind $\left(f_{1}, f_{2}, a\right)=0$ então existem $\left(f_{1}^{\prime}, f_{2}^{\prime}\right) \simeq\left(f_{1}, f_{2}\right)$ e $U$ aberto com $a \in U$ tais que:

(i) $\operatorname{Coin}\left(f_{1}, f_{2}\right) \cap \bar{U}=\{a\}$;

(ii) $f_{i}^{\prime}=f_{i}$ em $x \backslash U, \quad i=1,2$;

(iii) $\operatorname{Coin}\left(f_{1}^{\prime}, f_{2}^{\prime}\right) \cap U=\emptyset$.

Lema 3.5 Seja $f: X^{\prime} \rightarrow Y$ uma aplicação entre variedades n-dimensionais $(n \geq 2)$ conexas, compactas, orientáveis. triangularizáveis e sem bordo. Dado $\varepsilon>0$ existe $f^{\prime}$ : $X \rightarrow Y$ uma aplicação E-homotópica a f com, no máximo. um ponto de coincidência com $f$.

Demonstração: [Schimer 3.pg 32].

Teorema 3.6 Seja $f: X \rightarrow Y$ uma aplicaçảo entre duas variedades $n$-dimensionais $(n \geq 2)$ conexas. compactas, orientáveis, triangularizáveis $\epsilon$ sem bordo. Se $A \neq \emptyset \in$ um fechado em X então existe um par $\left(f_{1}, f_{2}\right) \operatorname{com} f_{1} \simeq f \simeq f_{2}$ tal que $\operatorname{Coin}\left(f_{1}, f_{2}\right)=$ .t. 
Demonstração: Escolhemos ह e. pelo lema 3.5. existe $f^{\prime}: X^{\prime} \rightarrow Y$. E-homotópica à $f$ com no máximo um ponto de coincidência com $f$.

Se $C \operatorname{oin}\left(f, f^{\prime}\right)=\emptyset$ escolhemos $a \in \mathrm{X}$ e um $\eta>0 \operatorname{com} f(\bar{C}(a \cdot \eta)) \cap f^{\prime}(\bar{C}(a \cdot \eta))=\emptyset$ e de modo que exista $p \in Y \backslash\left(f(\bar{U}(a, \eta)) \cup f^{\prime}(\bar{U}(a, \eta))\right) \operatorname{com}\left(f\left(\overline{L^{C}}(a, \eta)\right) \cup f^{\prime}(\bar{U}(a, \eta))\right) \subset$ ¿ $(p, \Sigma)$.

Tomamos $\gamma, \gamma^{\prime}: I \rightarrow U(p, \Xi)$ curvas em $Y \operatorname{com} \gamma(0)=f(a), \gamma^{\prime}(0)=f^{\prime}(a)$. $\gamma(1)=\gamma^{\prime}(1)=p$ e $\gamma(t)=\gamma^{\prime}\left(t^{\prime}\right)$ somente se $t=t^{\prime}=1$.

Sendo $\zeta: \bar{U}(a, \eta) \backslash\{a\} \rightarrow \partial \bar{U}(a, \eta)$ e $\zeta_{\frac{1}{2}}: \bar{U}\left(a, \frac{\eta}{2}\right) \backslash\{a\} \rightarrow \partial \bar{U}\left(a, \frac{\eta}{2}\right)$ as projeçòes com centro em $a$. Definimos $f_{1}^{\prime}, f_{2}^{\prime}: X \rightarrow Y^{\prime}$ tais que

$$
f_{1}^{\prime}(x)= \begin{cases}f(x) & \text { se } \quad x \in X \backslash \bar{U}(a, \eta) \\ t \cdot f(\zeta(x))+(1-t) \cdot f(a) & \text { se } \quad x=t \zeta(x)+(1-t) \zeta_{\frac{1}{2}}(x) 0 \leq t \leq 1 \\ \gamma(t) & \text { se } \quad x=t \zeta_{\frac{1}{2}}(x)+(1-t) a \leq t \leq 1\end{cases}
$$

$\mathrm{e}$

$$
f_{2}^{\prime}(x)= \begin{cases}f^{\prime}(x) & \text { se } x \in X \backslash \bar{U}(a, \eta) \\ t \cdot f^{\prime}(\zeta(x))+(1-t) \cdot f^{\prime}(a) & \text { se } x=t \zeta(x)+(1-t) \zeta_{\frac{1}{2}}(x) 0 \leq t \leq 1 \\ \gamma(t) & \text { se } x=t \zeta_{\frac{1}{2}}(x)+(1-t) a \leq t \leq 1 .\end{cases}
$$

Se $\operatorname{Coin}\left(f, f^{\prime}\right) \neq \emptyset$ tomamos $a=\operatorname{Coin}\left(f, f^{\prime}\right)$ e fazemos $f_{1}^{\prime}=f$ e $f_{2}^{\prime}=f^{\prime}$.

Temos assim que $f_{1}^{\prime}$ é $\varepsilon$-homotópica à $f_{2}^{\prime}$ e $\operatorname{Coin}\left(f_{1}^{\prime}, f_{2}^{\prime}\right)=\{a\}$.

Escolhemos $a_{0} \in A$ e, usando o lema 3.2 obtemos $f_{1}^{\prime \prime}$ e $f_{2}^{\prime \prime} \operatorname{com} \operatorname{Coin}\left(f_{1}^{\prime \prime}, f_{2}^{\prime \prime}\right)=\left\{a_{0}\right\}$. Da demonstração de 3.2 temos que $f_{1}^{\prime \prime}$ é $\varepsilon$-homotópica à $f_{2}^{\prime \prime}$.

Se $|K|$ e $|L|$ sào triangularizaçòes de $X$ e $Y$ respectivamente. Podemos escolher $\varepsilon<\operatorname{mesh}(L)$. Dessa forma: $f_{1}^{\prime \prime} \varepsilon$-homotópica à $f_{2}^{\prime \prime}$ implica que $\overline{\sigma\left(f_{1}^{\prime \prime}(x)\right)} \overline{\overline{\sigma\left(f_{2}^{\prime \prime}(x)\right)}} \neq \emptyset$ para todo $x$ em $X$.

Fazendo $\delta$ igual ao diâmetro de $\left|K^{\prime}\right|$ e $t(x)=\frac{d(x, A)}{\delta}$ definimos $f:\left|K^{\prime}\right| \rightarrow|L|$ dada por

$$
f_{1}(x)=h\left(f_{1}^{\prime \prime}(x), f_{2}^{\prime \prime}(x), t(x)\right)
$$

onde $h$ é dada pelo lema 2.1.

Denotando $f_{2}^{\prime \prime}$ por $f_{2}$ temos que

$$
\operatorname{Coin}\left(f_{1}, f_{2}\right)=A \text { e } f_{1} \simeq f \simeq f_{2} .
$$


Teorema 3.7 Sejam X $X$ Y variedades n-dimensionais $(n \geq 3)$. orientáceis. compactas. coneras. sem bordo e triangularizáceis. Se $f_{1}, f_{2}$ são aplicaçòes de $\mathrm{X}$ em Y entào existem aplicaçóes $f_{1}^{\prime}, f_{2}^{\prime}: X \rightarrow Y$ homotópicas à $f_{1} \in f_{2}$ respectivamente com exatamente. $V\left(f_{1}, f_{2}\right)$ coincidências.

Demonstração: [Schimer 3,pg 36].

Teorema 3.8 Sejam X $\mathrm{X}$ e variedades $n$-dimensionais $(n \geq 3)$, orientáveis, compactas. conexas, sem bordo e triangularizáceis: $f_{1}, f_{2}: X \rightarrow Y$ aplicações com classes de coincidencia essenciais $\mathbb{F}_{l}, c_{1}, \ldots, c_{m}$ pontos de $X \in i_{1}, \ldots, i_{m}$ inteiros. Existe $\left(f_{1}^{\prime}, f_{2}^{\prime}\right) \simeq\left(f_{1}, f_{2}\right)$ com $\operatorname{Coin}\left(f_{1}^{\prime}, f_{2}^{\prime}\right)=\left\{c_{1}, \ldots, c_{m}\right\}$ e ind $\left(f_{1}^{\prime}, f_{2}^{\prime}, c_{k}\right)=i_{k}$ se, e somente se, $c_{k}$ pode ser reindexado $c_{j}$ com $1 \leq j=j(k) \leq m$ de modo que: ou $N\left(f_{1}, f_{2}\right)=0$ e $\sum_{j=1}^{m} i_{j}=0$ ou existe uma sequência de inteiros $m_{0}, \ldots, m_{N\left(f_{1}, f_{2}\right)}$ com $0=m_{0}<m_{1}<. .<m_{N\left(f_{1}, f_{2}\right)}=m$ que satisfa $\sum_{j=m_{l-1}+1}^{m_{l}} i_{j}=\operatorname{ind}\left(f_{1}, f_{2}, \mathbb{F}_{l}\right)$ para $l=1, \ldots, N\left(f_{1}, f_{2}\right)$.

Demonstração: $(\Rightarrow)$ Trivial.

$(\Leftarrow)$ Usando o teorema 3.7 obtemos $\left(g_{1}, g_{2}\right) \simeq\left(f_{1}, f_{2}\right)$ com exatamente $N\left(f_{1}, f_{2}\right)$ pontos de coincidência.

Se $N\left(f_{1}, f_{2}\right)=0$ o par $\left(g_{1}, g_{2}\right)$ é livre de coincidências. Usando a mesma construção feita na demonstração do teorema 3.6 obtemos $\left(g_{1}^{\prime}, g_{2}^{\prime}\right) \simeq\left(g_{1}, g_{2}\right) \operatorname{com} \operatorname{Coin}\left(g_{1}^{\prime}, g_{2}^{\prime}\right)=$ $\{a\}$ um ponto de $X^{\prime}$.

Através dos lemas 3.3 e 3.2 obtemos o par desejado.

Se $N\left(f_{1}, f_{2}\right)>0$ temos que $\operatorname{Coin}\left(g_{1}, g_{2}\right)=\left\{a_{1}, \ldots, a_{N\left(f_{1}, f_{2}\right)}\right\}$; usando o lema 3.3 "transformamos" cada $a_{l}$ em $m_{l}-m_{l-1}$ pontos de coincidência de um par $\left(g_{1}^{\prime}, g_{2}^{\prime}\right) \simeq$ $\left(g_{1}, g_{2}\right)$ (isso é feito de modo que os índices desses pontos sejam os $i_{j}$ desejados). Novamente com o lema 3.2 "colocamos" cada $b_{j} \in C$ oin $\left(g_{1}^{\prime}, g_{2}\right)$ em $c_{j}$, obtendo, assim. o par $\left(f_{1}^{\prime}, f_{2}^{\prime}\right)$.

O problema de localização de coincidências apresenta uma "face" nova quando comparado ao de localização de pontos fixos. Com que liberdade podemos localizar a imagem das coincidências de um par de aplicaçòes?

Nesse sentido apresentamos alguns resultados.

Lema 3.9 Sejam $f_{1}, f_{2}: X \rightarrow Y$ um par de aplicaçóes entre variedades n-dimensionais $(n \geq 2)$. conexas $\epsilon$ sem bordo. Se a é uma coincidência isolada de $f_{1}$ e $f_{2}$ então. 
dado $p \in Y$. existe $\left(f_{1}^{\prime}, f_{2}^{\prime}\right) \simeq\left(f_{1}, f_{2}\right) \operatorname{com} \operatorname{Coin}\left(f_{1}^{\prime}, f_{2}^{\prime}\right)=C \operatorname{oin}\left(f_{1}, f_{2}\right), f_{i}^{\prime}=f_{i} \epsilon m$ uma vizinhança de $\operatorname{Coin}\left(f_{1}, f_{2}\right) \backslash\{a\} \in f_{1}^{\prime}(a)=f_{2}^{\prime}(a)=p$.

Demonstração: Claramente existe em $Y^{*}$ um aberto $W^{*} \operatorname{com} \bar{W}$ homeomorfo, por $\theta$. à $B^{n}$ que contenha $f_{1}(a)$ e $p$ (podemos obter tal aberto tomando uma vizinhança suficientemente pequena de uma curva simples de $a$ até $p$ ).

Tomamos $U$ uma vizinhança de $a$ homeomorfa, por $\varphi$, ̀̀ $B^{n}(\varphi(a)=0)$, tal que $f_{1}(\bar{U}) \cup f_{2}(\bar{U}) \subset H^{\circ}$.

Usando a projeção central em $B^{n}$ obtemos que para cada $x \in U$ existe um único $x_{\partial} \in \partial U$ tal que $\varphi(x)=t_{x} \varphi\left(x_{\partial}\right)$ com $0<t_{x} \leq 1$.

Definimos:

$$
f_{i}^{\prime}(x)=\left\{\begin{array}{lll}
f_{i}(x) & \text { se } & x \in X \backslash U \\
\theta^{-1}\left(t_{x} \theta\left(f_{i}\left(x_{\partial}\right)\right)+\left(1-t_{x}\right) \theta(p)\right) & \text { se } & x \in U
\end{array}\right.
$$

$i=1,2$.

Claramente $f_{i}^{\prime}$ é homotópica à $f_{i}$ basta tomarmos

$$
H(x, t)=\theta^{-1}\left(t \cdot \theta\left(f_{i}(x)\right)+(1-t) \cdot \theta\left(f_{i}^{\prime}(x)\right)\right) .
$$

Como $f_{1}(x) \neq f_{2}(x) \forall x \in \partial(U)$ temos que $\operatorname{Coin}\left(f_{1}^{\prime}, f_{2}^{\prime}\right)=\operatorname{Coin}\left(f_{1}, f_{2}\right)$ e $f_{1}^{\prime}(a)=$ $f_{2}^{\prime}(a)=p$.

É interessante observar que não usamos a hipótese de $W$ ser aberto; bastava que as imagens de $U$ por $f_{1}$ e $f_{2}$ estivessem contidas em um conjunto $W^{\prime}$ homeomorfo à $B^{n} \operatorname{com} f_{i}(a)$ e $p$ contidos em $W^{\prime}$. Dessa forma a demonstração acima pode ser feita em casos mais gerais, por exemplo, se $X$ e $Y$ forem complexos simpliciais de mesma dimensào com $a, f_{i}(a), p$ contidos em simplexos maximais e $Y$ for $n$-dimensionalmente conexo.

Teorema 3.10 Sejam $X^{r} \in Y$ variedades $n$-dimensionais $(n \geq 2)$, coneras e sem bordo. Se $f_{1}, f_{2}: X \rightarrow Y$ é um par de aplicaçòts com Coin $\left(f_{1}, f_{2}\right)=\left\{a_{1}, \ldots, a_{m}\right\}$ então. dados $p_{1}, \ldots . p_{m} \in Y$ existem aplicaçòes $f_{1}^{\prime}, f_{2}^{\prime}: X \rightarrow Y$ tais que:

(i) $\left(f_{1}, f_{2}\right) \simeq\left(f_{1}^{\prime}, f_{2}^{\prime}\right)$;

(ii) $\operatorname{Coin}\left(f_{1}^{\prime}, f_{2}^{\prime}\right)=\operatorname{Coin}\left(f_{1}, f_{2}\right)$;

(iii) $f_{1}^{\prime}\left(a_{j}\right)=f_{2}^{\prime}\left(a_{j}\right)=p_{j}$ para $j=1, \ldots, m$. 
Demonstração: Basta usarmos repetidas vezes o lema 3.9 .

Usando o teorema acima podemos "completar" o teorema 3.8 de modo que a imagem de cada uma das coincidências $c_{j}$ seja qualquer ponto dado em $Y$.

Lema 3.11 Sejam $|\mathrm{X}|$ e $|Y|$ poliedros conexos de dimensão $n \geq 2$. $|Y|$ compacto. Se $f:|X| \rightarrow|Y| e^{\prime}$ uma aplicaçào então existe $f^{\prime} \simeq f$ sobrejetora.

Demonstração: Tomamos $a \in\left|X^{r}\right| \operatorname{com} \sigma(a)$ de dimensão $n$, de modo que exista $\varepsilon>0 \operatorname{com} \bar{U}(a, \Xi) \subset|\sigma(a)|$.

Seja $\uparrow: I \rightarrow|Y|$ uma curva de $f(a)$ até $a^{\prime}, \operatorname{com} \sigma\left(a^{\prime}\right)$ de dimensão $n$ e $\eta>0$ tal que $\bar{U}\left(a^{\prime}, \eta\right) \subset\left|\sigma\left(a^{\prime}\right)\right|$.

Definimos $f_{1}:|X| \rightarrow|Y|$ por

$$
f_{1}(x)= \begin{cases}f(x) & \text { se } x \in|X| \backslash U(a, \Sigma) \\ f\left((1-t) x_{\varepsilon}+t a\right) & \text { se } x=(1-t) x_{s}+t x_{\frac{s}{2}} .0 \leq t \leq 1 \\ \gamma(t) & \text { se } x=(1-t) x_{\frac{\varepsilon}{2}}+t a .0 \leq t \leq 1\end{cases}
$$

onde $x_{\varepsilon}$ e $x_{\frac{\varepsilon}{2}}$ são, respectivamente, a imagem da projeção com centro $a$ em $\partial U(a, \varepsilon)$ e $\partial U\left(a, \frac{\varepsilon}{2}\right)$ (essa notação aparecerá outras vezes).

Dessa forma $f_{1}(a)=a^{\prime}$.

Existe $\varepsilon_{1}>0$ tal que $U\left(a, \varepsilon_{1}\right) \subset f^{-1}\left(U\left(a^{\prime}, \frac{\eta}{2}\right)\right)$.

Sendo $\varphi$ um homeomorfismo entre $\bar{U}\left(a, \frac{s_{1}}{2}\right)$ e $\bar{U}\left(a^{\prime}, \frac{\eta}{2}\right)$, definimos $f_{2}:\left|X^{\prime}\right| \rightarrow|Y|$ satisfazendo

$$
f_{2}(x)= \begin{cases}f_{1}(x) & \text { se } x \in|X| \backslash U\left(a, \varepsilon_{1}\right) \\ \varphi(x) & \text { se } x \in \bar{U}\left(a, \frac{s_{1}}{2}\right) \\ (1-t) f_{1}(x)+t \varphi(x) & \text { se } x=(1-t) x_{s_{1}}+t x_{\frac{s_{1}}{2}} \quad 0 \leq t \leq 1\end{cases}
$$

Se $\sigma_{1}, \ldots, \sigma_{m}$ são todos os simplexos maximais de $Y$, escolhemos $b_{1}, \ldots, b_{m}$ em $|Y|$ de modo que $b_{i} \in\left|\sigma_{i}\right|$. Tomamos $a_{1}, \ldots a_{m} \in U\left(a^{\prime} . \frac{\eta}{2}\right)$ e $\rho>0$ satisfazendo:

(i) $U\left(a_{i}, \rho\right) \subset U\left(a^{\prime}, \frac{\eta}{2}\right)$;

(ii) $\bar{U}\left(a_{i}, \rho\right) \cap \bar{U}\left(a_{j}, \rho\right)=\emptyset$ se $i \neq j$,

e $\gamma_{i}$, curvas de $a_{i}$ até $b_{i}$. 
Sendo $\psi_{i}$ uma aplicaçào sobrejetora de $\bar{L}\left(a_{i}, \frac{p}{4}\right)$ em $\overline{\left|\sigma_{i}\right|}$. com $\iota_{i}\left(a_{i}\right)=b_{i}$. usando a projeçào com centro em $a_{i}$, definimos $\alpha_{i}: \bar{L}\left(a_{i}, \rho\right) \rightarrow Y$ por

$$
\alpha_{i}(x)= \begin{cases}(1-t) x_{\rho}+t a_{i} & \text { se } x=(1-t) x_{\rho}+t x_{\frac{3 \rho}{4}} 0 \leq t \leq 1 \\ \hat{i}_{i}(t) & \text { se } x=(1-t) x_{\frac{3 \rho}{4}+t x_{\frac{\rho}{2}}} 0 \leq t \leq 1 \\ (1-t) b_{i}+t \psi(x) & \text { se } x=(1-t) x_{\frac{\rho}{2}}+t x_{\frac{\rho}{4}} 0 \leq t \leq 1 \\ \psi_{i}(x) & \text { se } x \in \bar{U}\left(a_{i}, \frac{\rho}{4}\right) .\end{cases}
$$

Definimos então $f^{\prime}:|X| \rightarrow|Y|$ por

$$
f^{\prime}(x)=\left\{\begin{array}{lll}
f_{2}(x) & \text { se } & f_{2}(x) \in|Y| \bigcup_{i=1}^{m} U\left(a_{i}, \rho\right) \\
\alpha_{i}\left(f_{2}(x)\right) & \text { se } & f_{2}(x) \in \bigcup_{i=1}^{m} U\left(a_{i}, \rho\right) .
\end{array}\right.
$$

Temos que $f^{\prime}$ é sobrejetora e, por construção, $f^{\prime} \simeq f$.

Teorema 3.12 Sejam $|X|$ e $|Y|$ poliedros conexos de dimensão $n \geq 2$, com $Y$ compacto do tipo $W . A \subset|Y|$ um fechado. $A \neq \emptyset$ se $\chi(|Y|) \neq 0$. Se $f:|X| \rightarrow|Y|$ é uma aplicação então existe um par $f^{\prime}, f^{\prime \prime}:|X| \rightarrow|Y|, f^{\prime} \simeq f \simeq f^{\prime \prime} \operatorname{com} f^{\prime}\left(\operatorname{Coin}\left(f^{\prime}, f^{\prime \prime}\right)\right)=$ A.

Demonstração: Usando o lema 3.11 obtemos $f^{\prime}:\left|X^{\prime}\right| \rightarrow\left|Y^{\prime}\right|$ sobrejetora, $f^{\prime} \simeq f$. Pelo teorema 2.9 existe $h:|Y| \rightarrow|Y| \operatorname{com} F i x(h)=A$ e, da demonstraçào de 2.9, temos que $h \simeq i d$. Assim definimos $f^{\prime \prime}:\left|X^{\prime}\right| \rightarrow|Y|$ com

$$
f^{\prime \prime}(x)=h \circ f^{\prime}(x) \text {. }
$$

Dessa forma $f^{\prime}\left(C_{\operatorname{oin}}\left(f^{\prime}, f^{\prime \prime}\right)\right)=. A$ e $f^{\prime} \simeq f \simeq f^{\prime \prime}$. 


\section{Bibliografia}

[Brown] Brown, Robert F. The Lefschetz Fixed Point Theorem. Scott, Foresman and Company; 1971.

[Dold 1] Dold. Albrecht. Fixed point index and fixed point theorem for Euclidean neighborhood retracts. Topology 4 (1965), 1-\$.

[Dold 2] Dold. Albrecht. Lectures Notes on Algebraic Topology. Spring-Verlag, 1980.

[Jiang] Jiang, Boju. Lectures on Nielsen Fixed Point Theory. Contemporary Mathematics, vol 14; 1982.

[Kiang] Kiang, Tsai-han. The Theory of Fixed Points Classes. Springer-Verlag; 1980.

[Oliveira] Oliveira, Edson de. Teoria de Nielsen para Coincidência e Algumas Aplicaçǒes. Tese de Doutorado, USP, 1987.

[Schimer 1] Schimer, Helga. Fixed point sets of polyhedra. Pacific. J. Math. 52 (1974), 221-226.

[Schimer 2] Schimer, Helga. Mappings of polyhedra with prescribed fixed points an fixed point indices. Pacific. J. Math. 63 (1976), 521-530.

[Schimer 3] Schimer, Helga. Mindestzahlen von Koinzidenzpunkten. J. Reine und Angrew. Mathematik 194 (1955), 21-39.

[Vick] Vick, James W. Homology Theory An Introduction to Algebraic Topology: Academic Press; 1973. 